Document downloaded from:

http://hdl.handle.net/10251/50028

This paper must be cited as:

Rovira Cardete, A.; Roda Buch, A.; Lewis, R.; Marshall, M. (2012). Application of Fastsim with variable coefficient of friction using twin disc experimental measurements. Wear. 274275:109-126. doi:10.1016/j.wear.2011.08.019.

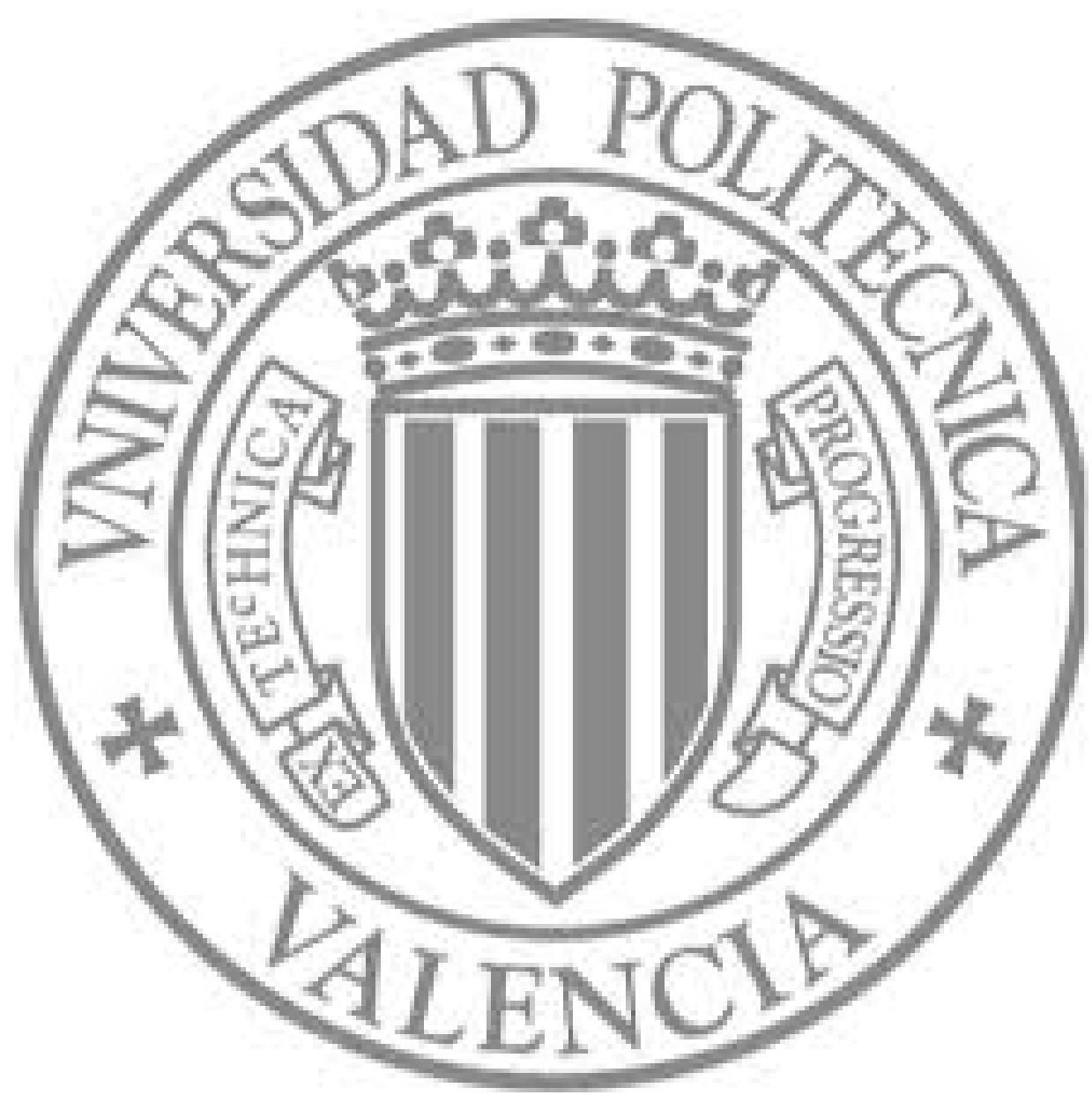

The final publication is available at

http://dx.doi.org/10.1016/j.wear.2011.08.019

Copyright Elsevier 


\title{
Application of Fastsim with variable coefficient of friction using twin disc experimental measurements
}

\author{
A. Rovira ${ }^{1 *}$, A. Roda ${ }^{1}$, R. Lewis ${ }^{2}$, M.B. Marshall ${ }^{2}$ \\ ${ }^{1}$ Universitat Politecnica de Valencia, CITV, C. Vera s/n, E46022 Valencia, Spain \\ ${ }^{2}$ University of Sheffield, Dep. of Mechanical Engineering, Mappin Street, S1 3JD Sheffield, UK \\ *Corresponding author: Tel. +34 963877007 Ext. 76263, fax: +34 96387 7629. E-mail address: \\ anrocar@mcm.upv.es (A. Rovira).
}

\begin{abstract}
In the field of railway simulation, it is a general assumption to consider the coefficient of friction as a known and constant value. This hypothesis is clearly not correct as friction is a consequence of the operating conditions (an output, not an input) and many factors can cause friction coefficient to change. In this paper, numerical algorithms based on the simplified theory of Kalker (Fastsim) and capable of modelling variable friction are studied and improved to match experimental measurements. Experimental creep curves from twin disc measurements are used to extract the friction parameters required by the numerical algorithms. Different friction functions are tested to correlate the effect of the contaminants and the pressure on the coefficient of friction.

Finally, two examples are shown to highlight the differences between modelling the wheel-rail contact with variable or constant coefficient of friction and their implications in the estimation of the interaction forces and wear.
\end{abstract}

KEYWORDS: Rail-wheel tribology, contact mechanics, rolling-sliding, creep forces, slip-dependent friction, wear modelling

\section{Introduction}

Today the use of simulation tools instead of testing is an increasing trend to demonstrate running safety and acceptability of new designs of railway vehicles [1]. Therefore, an appropriate model of the wheel-rail contact is crucial to get realistic results.

Among the theories developed to analyse the wheel-rail contact, Kalker has made the biggest contribution to the modelling of the tangential problem [2]. He evolved the simplified theory of rolling contact Fastsim [3] which processes the area of contact to get the tangential stress distribution within the contact patch. This algorithm was originally written for a constant value of the coefficient of friction (hereinafter COF). This, however, is never the case. Friction is a consequence of contact conditions and should not be used as an input to a model. All experimental data indicates varying friction with different contact conditions and as a result of changing environmental conditions. Examples of experimental work include Ohyama [4] who used a high speed large-sized rolling machine to study the discrepancies between theory and measurements. Magel et al. [5] with the aid of a rolling contact tribometer also studied the relation of some on-track measured curves with the theory. In this work he was able to measure creep curves at specific points on the track. This is ideally what is needed to feed into simulations.

Furthermore, some existing numerical models to solve the contact problem were adapted to get a better agreement with experimental measurements. Ertz et al. [6] introduced some modifications to the Shen, Hedrick and Elkins algorithm [7]. Knothe et al. [8] and Bucher et al. [9] conducted some creep curve calculations taking surface 
roughness into account and their effect on the shape of the curves. Polach [10-11] considered some coefficients in his wheel-rail contact theories to improve the conformity with experimental measurements. The calculation of the tangential problem with variable COF was treated by Nielsen et al. [12] who developed a model based on the exact theory of elasticity. Subsequently, Giménez et al. [2] and Piotrowski [13], developed an extension for Fastsim to model variable COF. In these three studies a friction function (or friction law) was included to describe the tangential behaviour of an element of the contact area.

Some applications such as the study of squeal noise or corrugation phenomena require a suitable model of the shape of the creep curve with the slope at saturated conditions [1416]. Therefore current models dealing with constant COF are not valid for these applications and new models as the mentioned before to model variable COF must be used.

In this paper, the extension of Fastsim to model the tangential contact with variable COF is used. Experimental curves coming from twin disc measurements are used to extract the friction parameters that define the friction functions. Furthermore, as a novelty in the use of the Fastsim algorithm with variable COF, a reduction in the initial slope is considered which is also identified from experimental creep curves. Thus, the Fastsim algorithm with variable COF and slope reduction is able to accurately reproduce the wheel rail tangential problem.

The paper is organised as follows: first of all, the general background of the consideration of variable friction in the Fastsim algorithm is presented; some definitions and the need of the slope reduction are also stated. This is followed with a description of the experimental method to measure the creep curves and the model of the twin disc contact. Afterwards, the algorithm used to identify the friction parameters and slope reduction is described. Subsequently, using some experimental creep curves, the parameters are identified for different surface contaminants and contact pressure. Then, this is applied to the actual wheel-rail geometry and two numerical examples are presented.

\section{Background}

\subsection{Fastsim algorithm with variable coefficient of friction}

In this section, the Fastsim algorithm developed by Kalker [3] is reviewed and the modifications required to consider the effect of the variable COF made by Giménez et al. [2] and Piotrowski [13] are described.

Taking the kinematic equation for the stationary state it is possible to calculate the nondimensional relative slip $\gamma=\left\{\gamma_{x} ; \gamma_{y}\right\}$ as a function of the rigid slip $\mathbf{w}=\left\{v_{x}-\varphi \cdot y ; v_{y}+\right.$ $\varphi \cdot \mathrm{x}\}$ and the displacements due to elastic deformations $\mathbf{u}=\left\{u_{x} ; u_{y}\right\}$, being $v_{x}, v_{y}$ and $\varphi$ the creepages.

$$
\boldsymbol{\gamma}=\mathbf{w}-\frac{\partial \mathbf{u}}{\partial x}
$$

Fastsim is based on the simplified theory of elasticity where the relation between the displacement due to elastic deformation of one point $\mathbf{u}(x, y)$ only depends on the 
tangential traction of this point $\mathbf{p}(x, y)=\left\{p_{x}(x, y) ; p_{y}(x, y)\right\}$, being $L$ the flexibility coefficient:

$$
\mathbf{u}(x, y)=L \mathbf{p}(x, y)
$$

Combining both equations and assuming that the tangential tractions are known, it is possible to obtain the non-dimensional slip as

$$
\boldsymbol{\gamma}(x, y)=\mathbf{w}(x, y)-L \frac{\partial}{\partial x} \mathbf{p}(x, y)
$$

There are two possibilities to compute the derivative in eq (3): to calculate it numerically, using finite differences, or to obtain the analytic expression of the derivative and evaluate this expression. The first one has the advantage that the function that describes the pressure distribution does not need to be known (this is the case if the normal pressure distribution is measured experimentally [17]). In the case that this expression is available, it has been shown that this second option provides smoother results for a rough discretisation. Furthermore, if there is an expression for the derivative, the algorithm is faster. This second option is used by Giménez et al. [2]. and Piotrowski [13], and is the option implemented in this work.

The solution of the tangential problem is obtained with the integration of equation (3) within the area of contact. This formula is used together with Coulomb's law to define the adhesion/slip regions. A value of the COF is assumed that depends on the relative slip $\gamma$ :

$$
\mu=\mu(\gamma)
$$

To evaluate the slip, different methods are used by the authors. On the one hand, Giménez et al. [2] developed an approximate formula to calculate the modulus of the slip:

$$
\gamma(x, y)=|\gamma(x, y)|=-\mathbf{w} \cdot\left\{\begin{array}{l}
\cos \left(\alpha_{1}\right) \\
\sin \left(\alpha_{1}\right)
\end{array}\right\}+L\left(\frac{\partial \mu(\gamma)}{\partial \gamma} p_{z}(x, y)+\mu(\gamma) \frac{\partial p_{z}(x, y)}{\partial x}\right)
$$

where $\alpha_{1}$ is the angle between the tangential stress vector and the $x$-direction. Substituting the analytical expressions of $\mu(\gamma), p_{z}(x, y)$ and their derivatives in the previous equation and iterating the formula, the modulus of the slip can be calculated. Fig. 1 (a) shows the tangential traction on a strip of the area calculated with this method.

On the other hand, Piotrowski [13] used the frictional power density to calculate the slip. In his paper, two families of methods are distinguished depending on how the COF in the slip area is recovered from (4): the methods with recuperation of static friction and the methods without recuperation of static friction. Piotrowski discards the former because it leads to a stick-slip instability in the contact area and there is no experimental evidence of this behaviour. Fig. $1(b)$ and $(c)$ show the results of the tangential traction calculated following the two methods from Piotrowski.

This work follows the algorithm developed by Giménez et al. [2] because it gets smoother results with a small number of elements. Nevertheless, the same process of 
parameter identification could be applied for the method without recuperation described by Piotrowski [12].

(a) $p_{x}$ calculated by Gimenez et al.

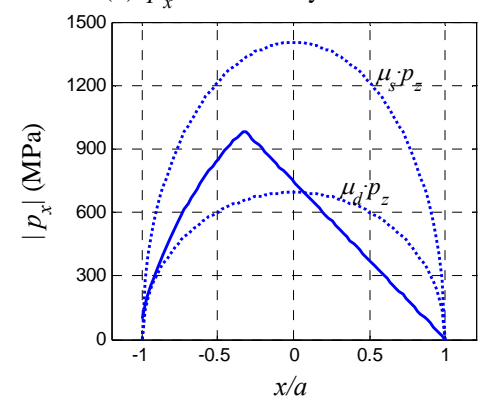

(b) $p$ Piotrowski with recuperation

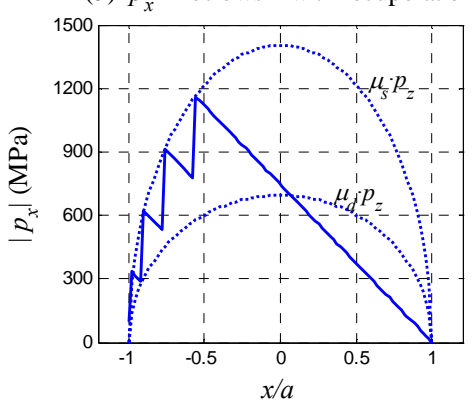

(c) $p$ Piotrowski w/o recuperation

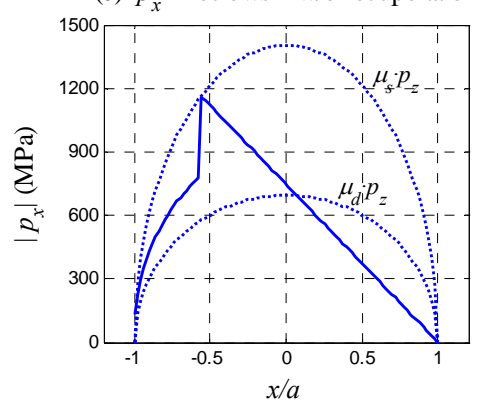

Fig. 1. Pressure distribution $p_{x}$ calculated for a strip under different hypothesis. $\mu_{s}$ and $\mu_{d}$ are the static and the dynamic value of the COF, respectively.

\subsection{Friction functions}

The friction function, or friction law, describes the relation between the nondimensional slip and the coefficient of friction (4). This relation is expressed locally for a point that belongs to the area of contact. Fig. 2 illustrates a diagram of a friction law with a constant COF and with a variable COF as a function of the slip $\gamma$ and their corresponding creep curves. This law cannot be directly measured, but can be estimated based on the tribology of the contact surfaces or identified from experimental measurements, as done in this paper.
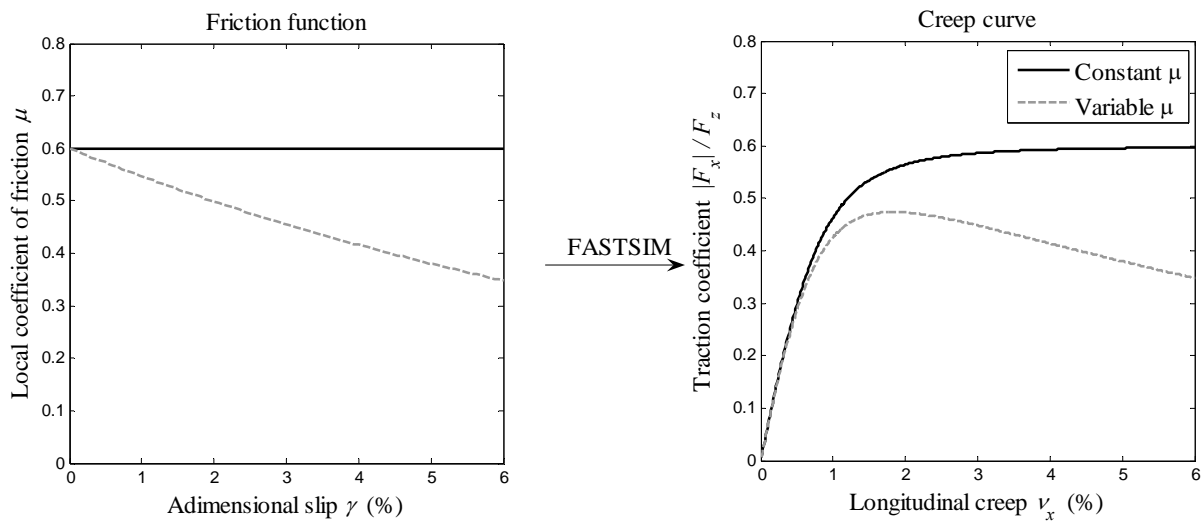

Fig. 2. Friction functions and their corresponding creep curves.

Analysing the creep curves (Fig. 2-right), two regions can be distinguished. The first region (below $0.7 \%$ approximately) shows a linear behaviour with a constant slope. This is due to the fact that the area is in adhesion, and the relation between the displacements and the stresses is linear (see equation (2)). This region is followed by the saturation of the area of contact. The second region corresponds with high values of creepage. In this region, the creep curves resemble their corresponding friction functions $[12,13]$. 
Three different models for the friction function are used in this paper:

- $\mathrm{f}_{1 \mathrm{a}}(\gamma)$, an exponential law that depends on three parameters: a static coefficient of friction $\mu_{o}$, a ratio of limit friction coefficient $\alpha$, and a parameter $\beta$ related to the curvature of the friction function. This function is used in $[11,13]$.

$$
\mathrm{f}_{1 a}(\gamma)=\mu_{o}\left[\alpha+(1-\alpha) \mathrm{e}^{-\beta \gamma}\right]
$$

- $\mathrm{f}_{1 \mathrm{~b}}(\gamma)$, a piecewise linear approximation for the previous formula. It also depends on three parameters: a static value of $\operatorname{COF} \mu_{s}$, a dynamic value $\mu_{d}$ and a threshold $\gamma_{c}$ as used in [2].

$$
\mathrm{f}_{1 b}(\gamma)=\left\{\begin{array}{cc}
\mu_{s}-\frac{\mu_{s}-\mu_{d}}{\gamma_{c}} \gamma & \text { if } 0 \leq \gamma \leq \gamma_{c} \\
\mu_{d} & \text { if } \gamma_{c}<\gamma
\end{array}\right.
$$

- $\mathrm{f}_{2}(\gamma)$, an exponential function which depends on four parameters: a static value for the COF $\mu_{s}$, a dynamic one $\mu_{d}$ and their corresponding decaying rates $\alpha$ and $\beta$, respectively.

$$
\mathrm{f}_{2}(\gamma)=\mu_{s} \mathrm{e}^{-\alpha \gamma}+\mu_{d}\left(1-\mathrm{e}^{-\beta \gamma}\right)
$$

The shape of the friction functions for standard values of their parameters is shown in Fig. 3 . On the plot on the left it can be seen how $\mathrm{f}_{1 \mathrm{~b}}$ approximates $\mathrm{f}_{1 \mathrm{a}}$. Both models will be tested under the same conditions to evaluate the differences between them. If there is a good correspondence between both functions, $\mu_{o} \approx \mu_{s}$ and $\alpha \cdot \mu_{o} \approx \mu_{d}$. On the right of Fig. 3 the friction function $f_{2}$ is plotted. It has an extra parameter to allow more flexibility when defining a friction function. The analytical derivative of those functions can easily be obtained for the inclusion in eq (5) to calculate the slip.

In the definition of the creep curves, the static COF is constrained to be greater or equal than the dynamic COF [18]. The examples on Fig. 3 meet this requirement regardless the shape of the curve for high values of slip. $f_{1 a}$ and $f_{1 b}$ are used to model the typical case of a decreasing creep curve after saturation. To model an increasing creep curve after saturation, $\mathrm{f}_{2}$ must be used.
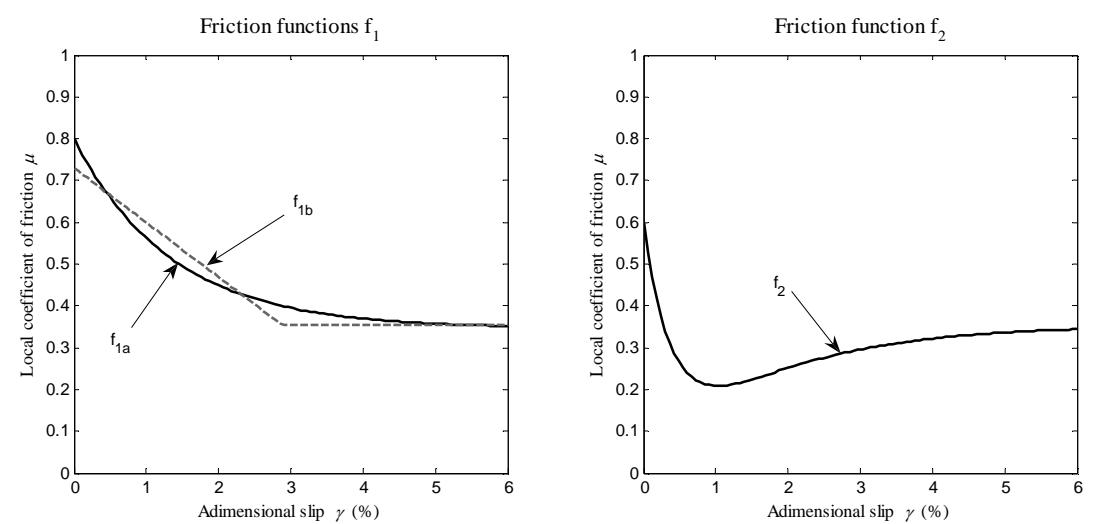

Fig. 3. Models for the friction functions $\mathrm{f}_{1 \mathrm{a}}$ and $\mathrm{f}_{1 \mathrm{~b}}$ (left) and $\mathrm{f}_{2}$ (right). 


\subsection{Reduction of the initial slope}

The initial slope depends on the flexibility coefficient $L$ (equation (2)). According to Kalker, this coefficient depends on the size and geometry of the contact and on the material properties [3], and is valid for scrupulously clean [5] and smooth surfaces [6-9] (with no asperities). Fig 4- $a$ ) shows the tangential contact modelled by Fastsim, for clean and smooth surfaces. The value of the tangential stiffness is $1 / L$.

On the other hand, Fig. 4- $b$ ) shows a real case where the effect of surface roughness (asperities) and the presence of a third body (contaminant) are considered. These two effects can be modelled as two serial springs, where the equivalent stiffness (inverse of the flexibility) is reduced with respect to the case with no asperities and clean surfaces:

- The asperities are taken into account with a reduced value of stiffness $\left(1 / L_{a}<\right.$ $1 / L$ ) or with a scaling factor $k_{a}$ which reduces the stiffness of the steel-steel contact (the subindex $a$ stands for asperity)

- The effect of contamination is also accounted with a reduced value of stiffness $\left(1 / L_{c}<1 / L\right)$ or with a scaling factor $k_{c}$ to reduce the stiffness of the steel-steel contact (the subindex $c$ stands for contamination), as in the previous case.

The equivalent flexibility can be computed as in [19]:

$$
L^{*}=L_{a}+L_{c}=\frac{L}{k_{a}}+\frac{L}{k_{c}}=\frac{L}{k}
$$

where $0<k \leq 1$ and it is calculated as $1 / k=1 / k_{a}+1 / k_{c}$.

These two effects are considered with a scaling factor $k$, which reduces the value of the theoretical stiffness.

a)

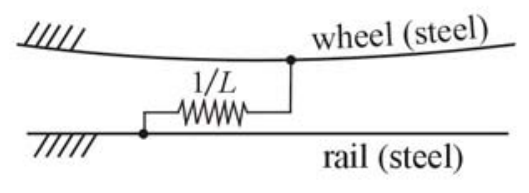

b)

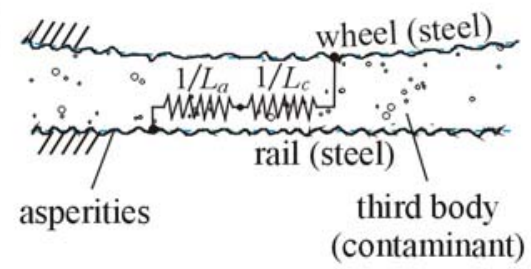

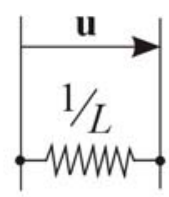

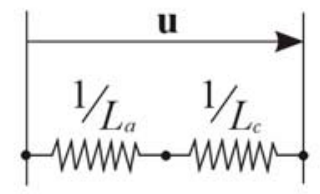

Fig. 4. Schematic and rheological model of the tangential contact for $a$ ) smooth and clean surfaces and $b$ ) rough and contaminated surfaces.

\section{The twin disc contact}

In this section the experimental procedure to obtain the creep curves used to extract the friction parameters and the wear data to construct the wear laws is briefly described. 
Data was acquired in a twin-disc machine where the contact geometry and the rollingsliding conditions are close to the actual in the wheel/rail contact. In the contact patch between the two rolling discs, there is an adhesion region in the leading edge, and a slip region in the trailing edge like in the wheel-rail contact. Furthermore, the normal pressure, the creepage (only longitudinal) and the shear stress are similar to the ones present in the wheel-rail contact.

Secondly, the hypotheses used to model the twin-disc contact problem are stated. This allows characterising the twin-disc contact and using the parameters identified from experimental measurements.

\subsection{SUROS twin-disc rig}

The experimental data was obtained in the SUROS (Sheffield University ROlling Sliding) twin-disc rig. A detailed description of roller rig is given in [20]. Fig. 5 shows a schematic representation of the test discs running on the roller rig. The discs are hydraulically loaded together and driven at controlled rotational speed by independent electric motors in order to get the desired value of creepage. The liquid feed (water, oil, friction modifier) is applied on the upper disc (rail) before the discs are loaded together. The contaminant based on particles (leaves, sand) is applied at the contact with the aid of a chute.

Discs were cut from rail and wheel tyres with a diameter $47.0 \mathrm{~mm}$ and a width of 10 $\mathrm{mm}$ [20]. Each point of the creep curve was obtained with a new pair of wheel and rail discs. The curve is measured with a steady state method, so when the response is stabilised, the value of the torque is measured and the traction coefficient is calculated. A comprehensive explanation about the experimental procedure can be found in [21].

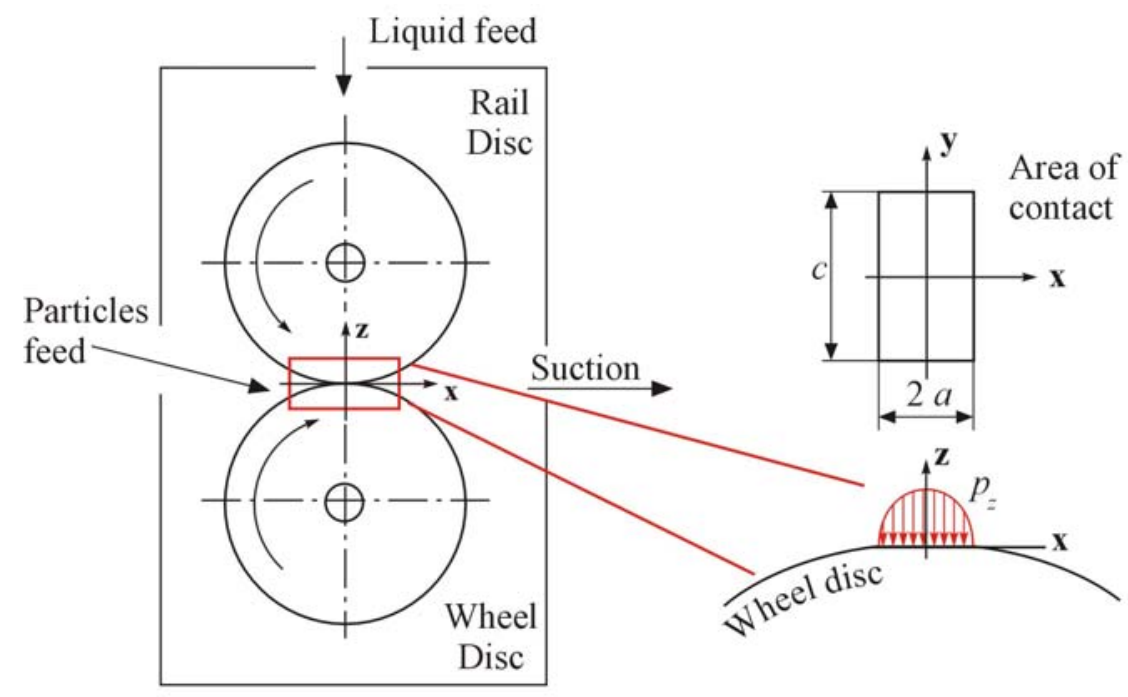

Fig. 5. Schematic diagram of the twin disc roller rig and area of contact generated by the two cylinders. 


\subsection{Modelling of the twin disc geometry}

The twin disc contact is modelled under the following hypotheses:

- The normal problem is considered as a two dimensional contact of cylindrical bodies which are rolling along their main axes. The contact is made over a long strip of width $c$ and length $2 \cdot a$ where $c>>2 a$ (Fig. 5-right). Hertz theory considers this case as the limit of an elliptical contact when $b$ is allowed to become large compared with $a$, being $a$ and $b$ the semi axes of the contact ellipse along the longitudinal and tangential directions [22].

- For the tangential problem, the Fastsim algorithm with variable COF is used. The value of the flexibility coefficient corresponding to the longitudinal direction, $L_{1}$, in equation (2) is obtained from the limit case of an ellipse where $b$ is much larger than $a$. Using the solution of the exact problem [23] for an elliptical area of contact, in the limit of an ellipse where $b$ is much greater than $a$ and $b \rightarrow c / 2$, the value of the elasticity parameter $L_{1}$ can be expressed as:

$$
L_{1}=\frac{4 a}{c_{11} G}
$$

where $G$ is the combined shear modulus for the wheel $(w)$ and rail ( $r$ ) materials $\left(1 / G=1 / 2\left(1 / G_{w}+1 / G_{r}\right)\right)$ and $c_{11}$ is the longitudinal coefficient of Kalker for the ratio of semiaxes $b / a$.

- Once obtained the theoretical gradient for small creepages which depends on $L_{1}$, this value is reduced using a factor $k$. This factor is chosen to fit the experimental data.

\section{Parameter identification from the creep curve}

As explained before, it is straightforward to obtain the creep curve from a friction function by the application of Fastsim [12]. To work the opposite way, i.e. to find the corresponding friction function for a given creep curve, is not possible. The approach used to do this is to find a friction function that generates a creep curve sufficiently equal to the desired one.

For a given friction model that depends on friction parameters and for a slope reduction, a full spectrum of creep curves are generated and it is chosen the creep curve that better approximates the experimental measurements. A relative error is calculated as the average error divided by a reference value:

$$
\varepsilon(\%)=\frac{1}{\mu_{\text {ref }}} \frac{\sum_{i=1}^{N}\left|e_{i}\right|}{N} \cdot 100
$$

where $\left|e_{i}\right|$ is the difference between the measured and calculated value of the creep curve, $N$ is the number of points measured for each creep curve and $\mu_{r e f}$ is the reference value of the coefficient of friction. For the value of $\mu_{\text {ref }}$ the traction coefficient for the point measured with the greatest creepage is taken. This error gives a qualitative idea of how good is the curve fit. 


\section{Results for the twin disc geometry}

In this section, using experimental creep curves measured with the SUROS test rig, friction parameters are identified. Data is taken from previous publications ([21, 24 and 25]). Firstly, the effect of surface contamination is studied. Second, the effect of the pressure on surface contamination is analysed, comparing creep curves for two different levels of pressure. Third, the effect of two different friction modifiers used as friction enhancers is investigated. Finally, a wear analysis for the twin disc contact is performed.

\subsection{Effect of surface contamination}

Creep curves are measured for dry discs and compared with the creep curves with the application of contaminants, such as water, oil, dry leaves and wet leaves. Dry and oil curves are taken from [24]. Wet, dry leaves and wet leaves curves are taken from [21]. Points are measured for a maximum pressure of $1500 \mathrm{MPa}$ on the discs, which is a typical of the actual wheel/rail contact, and for a nominal rotational velocity of $400 \mathrm{rpm}$ (which corresponds to a surface velocity of about $1 \mathrm{~m} / \mathrm{s}$ ). Data is collected for a range between 0.1 and $6 \%$ of creepage.

The experimental creep curves are shown in Fig. 6. Two families of curves can be distinguished:

- High adhesion curves: the traction coefficient is between 0.1 and 0.6. These curves exhibit a maximum and a falling traction coefficient beyond this value. This group is composed of the curves for dry and wet discs.

- Low adhesion curves: the traction coefficient for the range of creepage is less than 0.1. These curves show a slightly increasing shape for the whole range of creepage. This group is composed of the creep curves obtained for contamination with oil and with dry and wet leaves.
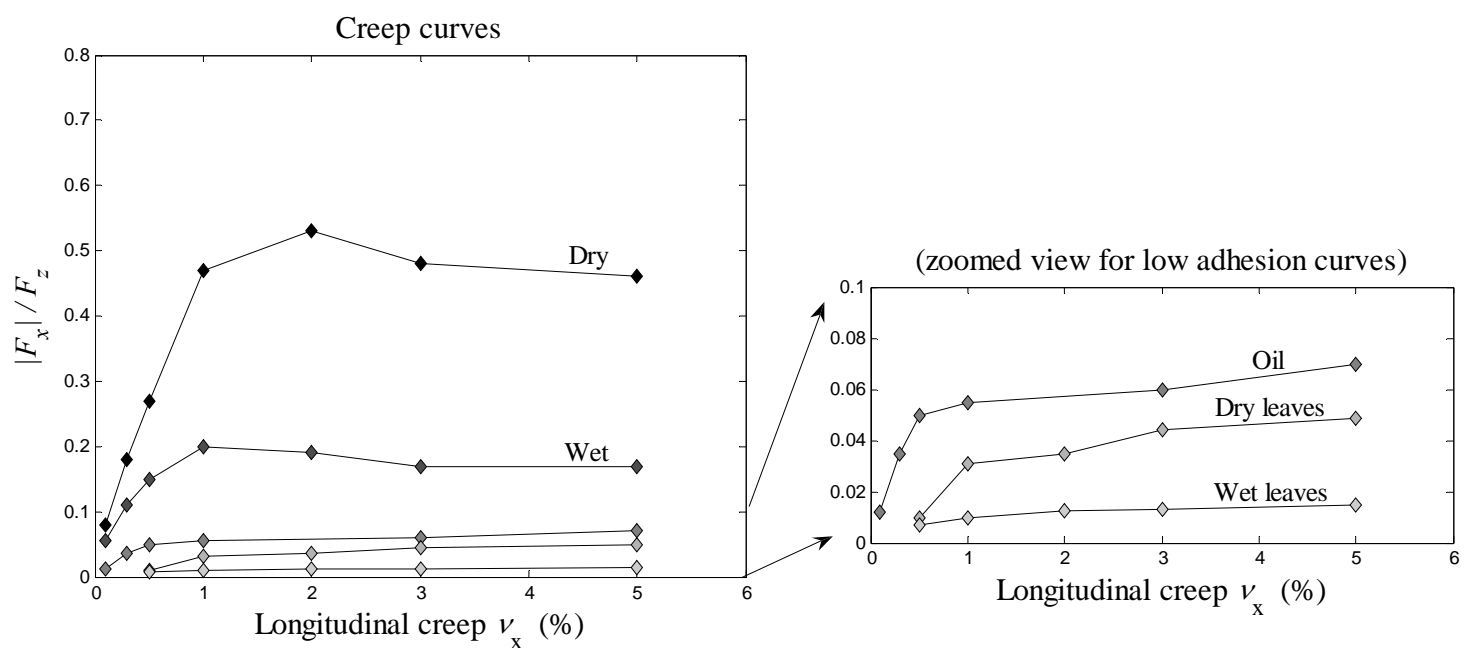

Fig. 6. Creep curves obtained on the SUROS roller rig. The right plot represents a zoomed view with the creep characteristic for the low adhesion curves. 
High adhesion curves are fitted using the models $\mathrm{f}_{1 \mathrm{a}}$ and $\mathrm{f}_{1 \mathrm{~b}}$ together with the slope reduction. They are characterised by a static value of the COF and a transition towards a dynamic COF. The friction functions and their corresponding creep curves are depicted in Fig. 7. The fit given by both models (exponential and piecewise functions) are very similar, which suggests that the use of the piecewise function is adequate. The values of the slope reduction, friction parameters and relative error are presented in Table 1 . For dry curves the coefficient $k$ is approximately equal to 1.00 . This means that the Kalker theory predicts well the contact rigidity and there is no slope reduction. This value is slightly greater than 1.00 and this is probably due to scatter in the measurement process. On the other hand, for wet surfaces the slope is multiplied by a factor approximately equal to 0.7. The values of the static and dynamic values of the COF can be seen on the Table. The relative error is less than $2 \%$.
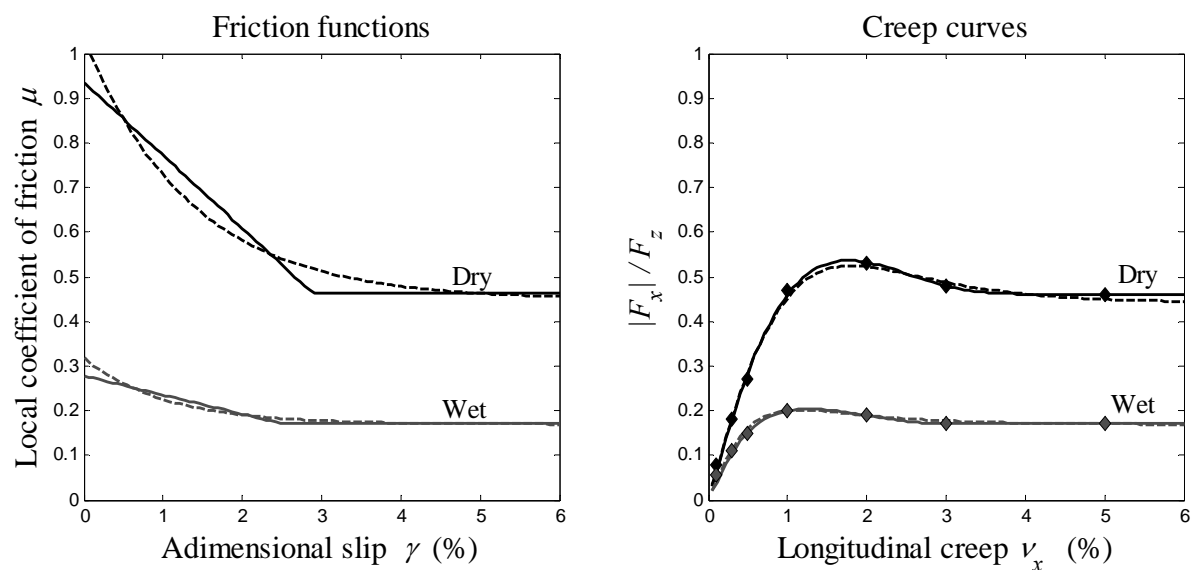

Fig. 7. Friction functions and creep curves for the high adhesion curves. Dash lines for $\mathrm{f}_{1 \mathrm{a}}$ and solid lines for $\mathrm{f}_{1 \mathrm{~b}}$.

Table 1. Parameters identified for the high adhesion curves for 1500 MPa using $f_{1 a}$ and $f_{1 b}$.

\begin{tabular}{|c|c|c|}
\cline { 2 - 3 } \multicolumn{1}{c|}{} & \multicolumn{2}{c|}{ Exponential function $\left(\mathrm{f}_{1 \mathrm{a}}\right)$} \\
\cline { 2 - 3 } \multicolumn{1}{c|}{} & Dry & Wet \\
\hline$k$ & 1.07 & 0.72 \\
\hline$\mu_{o}$ & 1.03 & 0.32 \\
\hline$\alpha$ & 0.43 & 0.53 \\
\hline$\beta$ & 74.29 & 97.05 \\
\hline$\varepsilon(\%)$ & 1.95 & 1.42 \\
\hline
\end{tabular}

\begin{tabular}{|c|c|c|}
\cline { 2 - 3 } \multicolumn{1}{c|}{} & \multicolumn{2}{c|}{ Piecewise function $\left(\mathrm{f}_{1 \mathrm{~b}}\right)$} \\
\cline { 2 - 3 } \multicolumn{1}{c|}{} & Dry & Wet \\
\hline$k$ & 1.06 & 0.65 \\
\hline$\mu_{s}$ & 0.94 & 0.28 \\
\hline$\mu_{d}$ & 0.46 & 0.17 \\
\hline$\gamma_{c}(\%)$ & 2.89 & 2.50 \\
\hline$\varepsilon(\%)$ & 1.46 & 0.36 \\
\hline
\end{tabular}

Low adhesion curves are fitted using the friction function $\mathrm{f}_{2}$. The friction functions and corresponding creep curves are depicted in Fig. 8. It is interesting to comment the shape of the friction functions, which resembles the Stribeck curve [18]. Dry leaves and wet leaves, behave as lubricants like the oil, but with a smaller COF. The friction function for wet leaves could also be modelled with a constant COF, due to the small variation of the creep curve in the range studied. The result of the parameter identification for the low adhesion curves is presented in Table 2. The relative error in the curve fit is less than $1 \%$. 

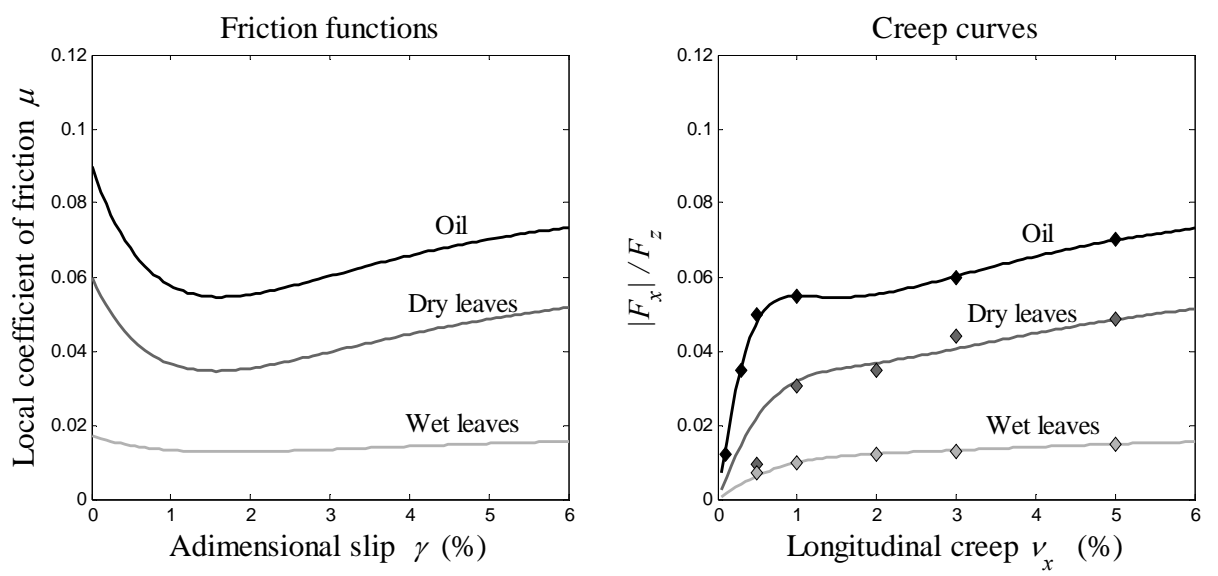

Fig. 8. Friction functions and creep curves for $1500 \mathrm{MPa}$ for the surfaces contaminated with oil, dry leaves and wet leaves.

Table 2. Parameters identified for the low adhesion curves for $1500 \mathrm{MPa}$ using the function $\mathrm{f}_{2}$.

\begin{tabular}{|c|c|c|c|}
\cline { 2 - 4 } \multicolumn{1}{c|}{} & \multicolumn{3}{c|}{ Exponential function $\left(\mathrm{f}_{2}\right)$} \\
\cline { 2 - 4 } \multicolumn{1}{c|}{} & Oil & Dry leaves & Wet leaves \\
\hline$k$ & 0.24 & 0.09 & 0.02 \\
\hline$\mu_{S}$ & 0.09 & 0.06 & 0.02 \\
\hline$\alpha$ & 107.78 & 111.43 & 80.00 \\
\hline$\mu_{d}$ & 0.08 & 0.06 & 0.02 \\
\hline$\beta$ & 41.11 & 32.86 & 40.00 \\
\hline$\varepsilon(\%)$ & 0.77 & 2.01 & 0.48 \\
\hline
\end{tabular}

The proposed method to identify the friction parameters from given creep curves is also applied for constant COF. The corresponding friction function is $\mathrm{f}(\gamma)=\mu$. Table 3 shows the results of the slope reduction $k$ and coefficient of friction $\mu$ for the surface conditions considered in this section and for $1500 \mathrm{MPa}$. As it is expected, the relative error is high compared to the error calculated for the different frictions functions used for variable COF (except for the case of dry leaves, where the error for constant COF is less than for variable COF).

Table 3. Parameters identified for $1500 \mathrm{MPa}$ for constant $\mu$.

\begin{tabular}{|c|c|c|c|c|c|}
\cline { 2 - 6 } \multicolumn{1}{c|}{} & \multicolumn{5}{c|}{ Constant $\mu$} \\
\cline { 2 - 6 } \multicolumn{1}{c|}{} & Dry & Wet & Oil & Dry leaves & Wet leaves \\
\hline$k$ & 1.12 & 0.84 & 0.22 & 0.04 & 0.02 \\
\hline$\mu$ & 0.49 & 0.17 & 0.06 & 0.05 & 0.02 \\
\hline$\varepsilon(\%)$ & 5.88 & 5.86 & 4.32 & 1.74 & 5.32 \\
\hline
\end{tabular}

\subsection{Effect of the pressure on the contaminants}

In this section the effect of the pressure on the creep curves is analysed. Two levels of pressure are considered for dry surfaces and with the presence of water and oil. The creep curves collected for a maximum Hertzian pressure of $1500 \mathrm{MPa}$ are taken from the previous section; the creep curves for $900 \mathrm{MPa}$ are extracted from [24].

For the high adhesion family (dry and wet) the friction models $\mathrm{f}_{1 \mathrm{a}}$ and $\mathrm{f}_{1 \mathrm{~b}}$ will be used; on the other hand, for the low adhesion family (oil), $\mathrm{f}_{2}$ will be used. The friction functions and corresponding creep curves are depicted in Fig. 9; the parameters of the 
curve fit are stated in Tables 4 and 5. The creep curve for dry contacts for $900 \mathrm{MPa}$ does not exhibit the typical shape with the maximum and the falling friction. Only one test was carried for each slip condition so it is possible the creep curve rises and then falls, but this may have been missed.
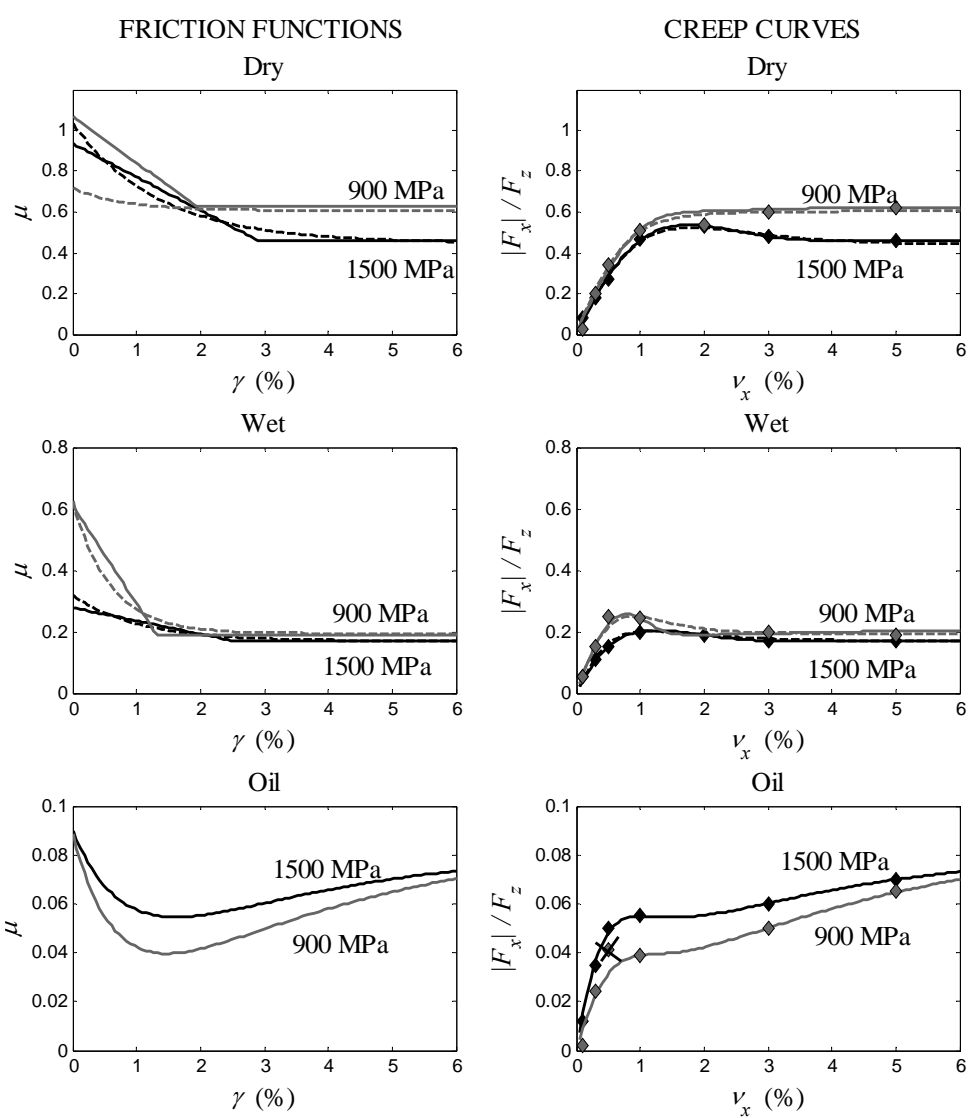

Fig. 9. Friction functions and creep curves fitted for dry, water and oil conditions for the two levels of pressure considered. For dry and wet, dash lines are for $\mathrm{f}_{1 \mathrm{a}}$ and solid lines for $\mathrm{f}_{1 \mathrm{~b}}$ (the third point of the curve for oil at $900 \mathrm{MPa}$ is eliminated).

Table 4. Parameters identified for Dry and Wet curves for 900 MPa using $\mathrm{f}_{1 \mathrm{a}}$ and $\mathrm{f}_{1 \mathrm{~b}}$.

\begin{tabular}{|c|c|c|}
\cline { 2 - 3 } \multicolumn{1}{c|}{} & \multicolumn{2}{c|}{ Exponential function $\left(\mathrm{f}_{1 \mathrm{a}}\right.$ ) } \\
\cline { 2 - 3 } \multicolumn{1}{c|}{} & Dry & Wet \\
\hline$k$ & 0.78 & 0.61 \\
\hline$\mu_{o}$ & 0.72 & 0.62 \\
\hline$\alpha$ & 0.84 & 0.31 \\
\hline$\beta$ & 129.41 & 170.59 \\
\hline$\varepsilon(\%)$ & 2.15 & 4.25 \\
\hline
\end{tabular}

\begin{tabular}{|c|c|c|}
\cline { 2 - 3 } \multicolumn{1}{c|}{} & \multicolumn{2}{c|}{ Piecewise function $\left(\mathrm{f}_{1 \mathrm{~b}}\right)$} \\
\cline { 2 - 3 } \multicolumn{1}{c|}{} & Dry & Wet \\
\hline$k$ & 0.72 & 0.59 \\
\hline$\mu_{s}$ & 1.06 & 0.61 \\
\hline$\mu_{d}$ & 0.62 & 0.19 \\
\hline$\gamma_{c}(\%)$ & 1.94 & 1.31 \\
\hline$\varepsilon(\%)$ & 3.37 & 4.30 \\
\hline
\end{tabular}


Table 5. Parameters identified for Oil for $900 \mathrm{MPa} u s i n g \mathrm{f}_{2}$.

\begin{tabular}{|c|r|}
\cline { 2 - 2 } \multicolumn{1}{c|}{} & Exp. F. $\left(\mathrm{f}_{2}\right)$ \\
\cline { 2 - 2 } \multicolumn{1}{c|}{} & Oil \\
\hline$k$ & 0.08 \\
\hline$\mu_{s}$ & 0.09 \\
\hline$\alpha$ & 140.00 \\
\hline$\mu_{d}$ & 0.09 \\
\hline$\beta$ & 26.29 \\
\hline$\varepsilon(\%)$ & 0.69 \\
\hline
\end{tabular}

As in the previous section, the slope reduction $k$ and the constant coefficient of friction $\mu$ are identified for a model of constant COF and for a pressure of $900 \mathrm{MPa}$ (Table 6). The relative error is greater than the one calculated for variable COF.

Table 6. Parameters identified for 900 MPa for constant $\mu$.

\begin{tabular}{|c|c|c|c|}
\cline { 2 - 4 } \multicolumn{1}{c|}{} & \multicolumn{3}{c|}{ Constant $\mu$} \\
\cline { 2 - 4 } \multicolumn{1}{c|}{} & Dry & Wet & Oil \\
\hline$k$ & 0.67 & 0.61 & 0.08 \\
\hline$\mu$ & 0.61 & 0.20 & 0.06 \\
\hline$\varepsilon(\%)$ & 4.10 & 13.02 & 10.34 \\
\hline
\end{tabular}

The variation of the pressure, for the other magnitudes kept constant, has two effects on the contact: on the normal problem, an increment on the pressure creates a variation on the size of the contact; on the tangential problem, the values of the friction parameters and slope reduction are different.

Regarding the normal problem, the relation between the semi-length of the area of contact and the maximum Hertzian pressure can be obtained [22]:

$$
a=2 p_{z_{o}} \frac{R^{*}}{E^{*}}
$$

where $R^{*}$ is the combined curvature of the two discs $\left(1 / R^{*}=1 / R_{w}+1 / R_{r}\right), E^{*}$ the combined Young's modulus of the wheel and rail materials $\left(1 / E^{*}=\left(1-\sigma^{2}\right) / E_{w}+(1-\right.$ $\left.\sigma^{2}\right) / E_{r}$ ) and $\sigma$ the Poisson's ratio.

Considering now the tangential problem, substituting equation (12) into (10), the flexibility coefficient for the longitudinal direction can be expressed as

$$
L_{1}=\frac{8 p_{z_{o}}}{c_{11} G} \frac{R^{*}}{E^{*}}
$$

As both relations depend linearly on the maximum Hertzian pressure $p_{z_{o}}$ and the differences between the creep curves for the two levels of pressure are relatively small, a linear transformation can be done to estimate the friction parameters and the slope reduction for the desired pressure. The results of the numerical calculations performed by Bucher et al. [26] also show a linear relation between the traction coefficient and the pressure. 
Using this technique, the different creep curves can be obtained for each value of maximum Hertzian pressure, with a linear transformation for the parameters of the friction function between 900 and $1500 \mathrm{MPa}$.

Fig. 10 shows the calculated creep curves for different values of pressure. They represent the surface plots for the creep curves which depend on two parameters: the maximum Hertzian pressure and the creepage. For a given value of maximum Hertzian pressure on the contact, the corresponding creep curve is taken as the slice of the surface plot for the value of the pressure. Similar surface plots can also be represented for the friction functions.
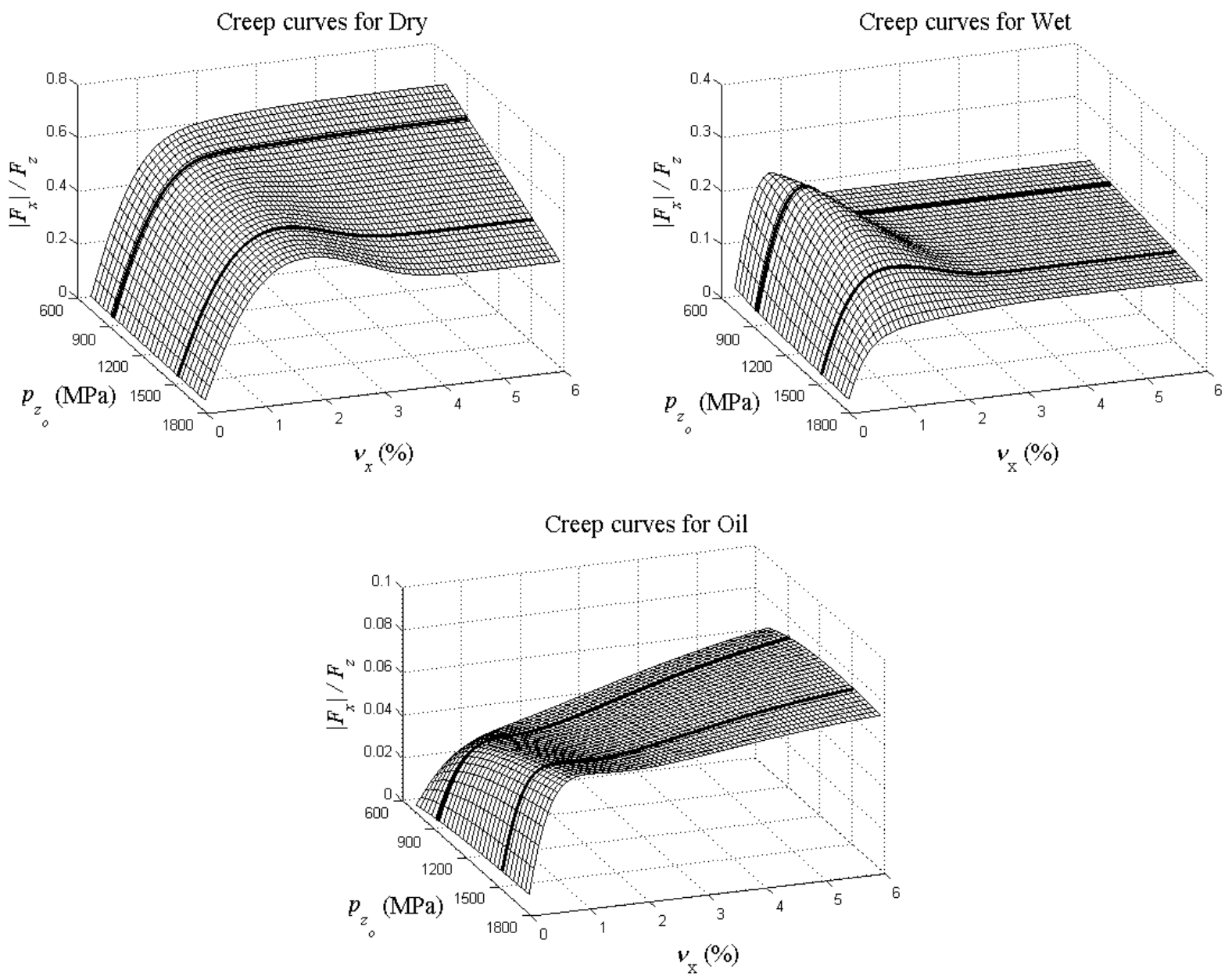

Fig. 10. Creep curves calculated for different values of pressure for Dry, Wet and Oil conditions. The curves for 900 and $1500 \mathrm{MPa}$ are plotted with a thicker line. For Dry and Wet it is used the friction function $\mathrm{f}_{1 \mathrm{~b}}$; for Oil, the friction function $\mathrm{f}_{2}$.

\subsection{Use of friction enhancers}

The third case studied corresponds to the use of friction enhancers which are one kind of the generic family of friction modifiers. Friction modifiers are used in the wheel/rail interface to improve one or more of these characteristics [25,27]:

- Eliminate the negative slope of the traction curve that is responsible for contact roll-slip instabilities, which may lead to the generation of squeal noise or corrugation (high positive friction modifiers). 
- Improve the adhesion (adhesion enhancers or traction enhancers).

- Reduce rolling contact fatigue.

- Reduce wear rate.

Friction modifiers are composed of engineered dry composite solids that are mixed with water and deposited on top of the rail in liquid form. When the water evaporates, the remaining thin dry film maintains an optimal intermediate level of friction [28].

In this section data is taken from [25] where the friction modifiers are used as adhesion enhancers (FE) to facilitate the traction and braking operation under poor adhesion conditions. Two friction modifiers are used, named FE A and FE B. FE A has been successfully tested in a train depot in Japan to overcome adhesion problems related to rainfall. FE B has extensively been used in autumn on the Dutch and British railways networks to mitigate adhesion problems mostly due to leaves and small amounts of water.

The creep curves are measured at $400 \mathrm{rpm}$ (which corresponds to a surface speed about $1 \mathrm{~m} / \mathrm{s}$ ) with a maximum Hertzian pressure of $1200 \mathrm{MPa}$ (there are no measurements taken for another level of pressure to allow interpolation as done in the previous section). FEs are applied in dry conditions on the wheel and rail discs. Both measured creep curves exhibit a positive slope throughout the creep range, therefore, the friction function $\mathrm{f}_{2}$ is used.
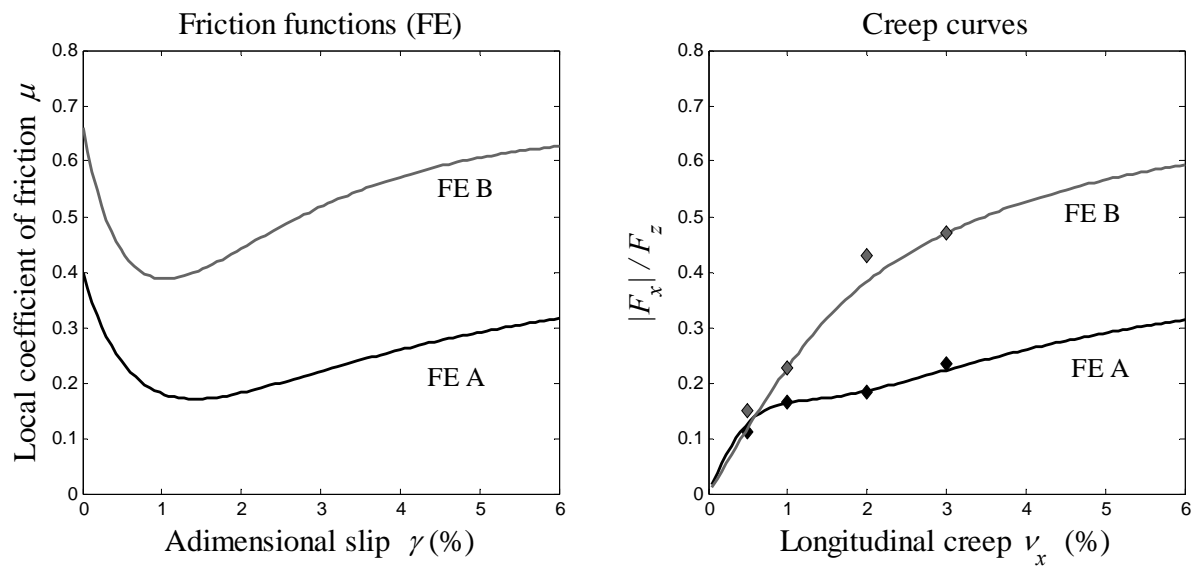

Fig. 11. Friction functions and creep curves for the adhesion enhancers.

The result of the parameter identification is shown in Fig. 11 and in Table 7. The shape of the friction functions for the FEs is quite similar to the one obtained for the oil. In fact, the positive characteristic given by a lubricant also reduces negative effects associated to the roll-slip instability and it was originally used to improve the wheel/rail contact. Nevertheless the low traction coefficient achieved with a lubricant (below 0.2) can compromise traction or braking operations. This does not happen with the use of a FE [28], which has a traction coefficient about 0.2 for FE A and 0.45 for FE B. 
Table 7. Parameters identified for the friction function $\mathrm{f}_{2}$ for the use of the tested friction enhancers on dry surfaces.

\begin{tabular}{|c|c|c|}
\cline { 2 - 3 } \multicolumn{1}{c|}{} & \multicolumn{2}{c|}{ Exponential function $\left(\mathrm{f}_{2}\right)$} \\
\cline { 2 - 3 } \multicolumn{1}{c|}{} & FE A & FE B \\
\hline$k$ & 0.44 & 0.32 \\
\hline$\mu_{s}$ & 0.40 & 0.66 \\
\hline$\alpha$ & 150.00 & 164.00 \\
\hline$\mu_{d}$ & 0.40 & 0.66 \\
\hline$\beta$ & 26.00 & 50.00 \\
\hline$\varepsilon(\%)$ & 1.63 & 2.60 \\
\hline
\end{tabular}

The slope reduction and friction parameters are also calculated for the case of constant COF (Table 8). As in the previous sections, the relative error calculated with the constant COF approach is greater than the relative error calculated for variable COF.

Table 8. Parameters identified for constant $\mu$.

\begin{tabular}{|c|c|c|}
\cline { 2 - 3 } \multicolumn{1}{c|}{} & \multicolumn{2}{c|}{ Constant $\mu$} \\
\cline { 2 - 3 } \multicolumn{1}{c|}{} & FE A & FE B \\
\hline$k$ & 0.34 & 0.36 \\
\hline$\mu$ & 0.21 & 0.55 \\
\hline$\varepsilon(\%)$ & 5.20 & 3.88 \\
\hline
\end{tabular}

\subsection{Wear analysis}

In this section the implications of the presence of contaminants on wear are analysed. The data of section 5.1 for dry, wet and oil surfaces is used. Wear is estimated from the energy dissipation in the contact patch. The latter is calculated with the index $T \gamma / A$. The University of Sheffield (USFD) wear law [29] is used to relate the energy dissipated and the amount of material removed.

The USFD law is based on twin disc experimental data and expresses wear as mass of worn material $(\mu \mathrm{g})$ per distance rolled $(\mathrm{m})$ per contact area $\left(\mathrm{mm}^{2}\right)$, following the equations in Table 9 and the plot in Fig. 12-(a). This function is obtained for R8T wheel and 900A rail materials in dry conditions. It is possible to take account of contaminants in the wheel/rail contact by simply changing the friction coefficient. This option may work fine for dynamic modelling. For application to wear modelling, this will change the $T \gamma$ value and therefore the wear rate by moving to a new point on the wear law. Nevertheless, a new wear law is needed. Fig. 12-(b) shows how the USFD law fits the trend for dry contacts. Wet contacts, however, do not follow the same curve (see the two points for wet contacts on Fig. 12-(b)).

Although wear laws are generally obtained only for dry conditions they are used in the presence of a surface contaminant, which is beyond the extend of their initial validation [30]. In this work the USFD wear law is used as a reference to show the estimation of wear assuming that the surfaces are dry and clean. 
Table 9. Wear regimes and coefficients for R8T wheel and 900A rail materials for dry contacts.

\begin{tabular}{|l|c|c|}
\hline \multicolumn{1}{|c|}{ Regime } & $T \gamma / A\left(\mathrm{~N} / \mathrm{mm}^{2}\right)$ & Wear Rate $\left(\mu \mathrm{g} / \mathrm{m} / \mathrm{mm}^{2}\right)$ \\
\hline $\mathrm{K}_{1}$ (mild) & $T \gamma / A \leq 10.4$ & $5.3 T \gamma / A$ \\
\hline $\mathrm{K}_{2}$ (severe) & $10.4<T \gamma / A \leq 77.2$ & 55.0 \\
\hline $\mathrm{K}_{3}$ (catastrophic) & $77.2<T \gamma / A$ & $55.0+61.9(T \gamma / A-77.2)$ \\
\hline
\end{tabular}

The energy dissipated in each element of the contact is calculated as the product of the tangential traction times the non-dimensional slip:

$$
\left.\frac{T \gamma}{A}\right|_{i}=\frac{T \gamma}{A}\left(x_{i}\right)=p_{x}\left(x_{i}\right) \cdot \gamma_{x}\left(x_{i}\right)
$$

A global value of the energy dissipated in the contact can be calculated as stated in (15), where $N$ is the number of elements of the contact patch, $\Delta x$ is the length of each element and $A_{s}$ is the area of slip per unit width. This value gives a global idea of the energy dissipated in the contact patch and the mechanisms involved in the wheel or rail wear.

$$
\frac{T \gamma}{A_{s}}=\left.\frac{1}{A_{s}} \sum_{i=1}^{N} \frac{T \gamma}{A}\right|_{i} \Delta x
$$

The USFD law can be implemented both locally and globally, considering the local stresses and slip within the contact patch or the global forces and creepages in the nominal point of contact [31]. In this paper the $T \gamma / A$ index and the USFD wear law are used in both ways.

a)

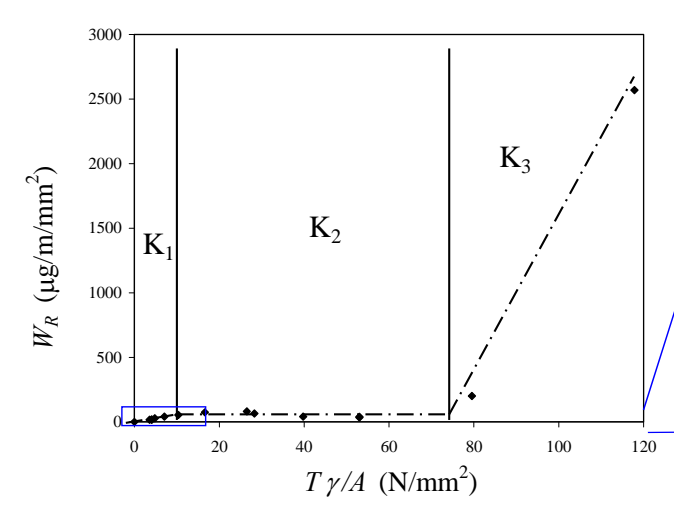

b)

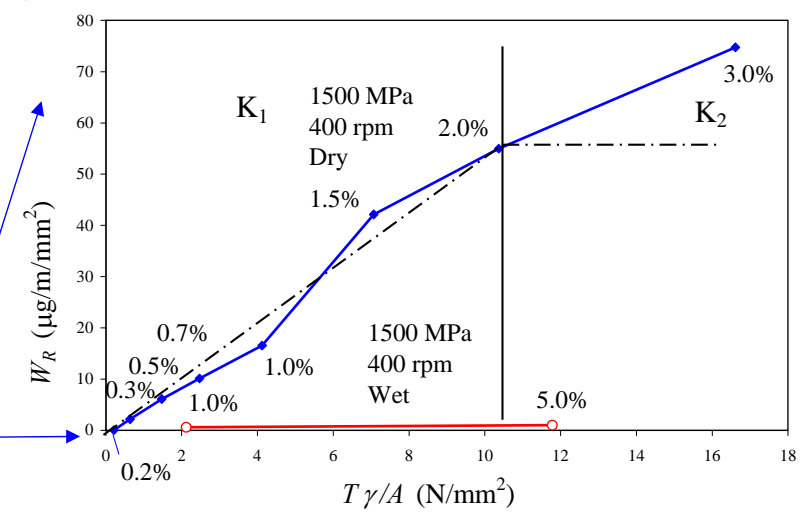

Fig. 12. (a) USFD wear function. (b) zoomed view of the USFD with points measured for dry and wet condition. The values on the plot are the longitudinal creepage (\%).

The tangential problem is studied under three different scenarios:

- (a) $v_{x}=0.5 \%$ : most of the area of contact is in adhesion. This corresponds to the linear region of the creep curve.

- (b) $v_{x}=2.0 \%$ : general case, with adhesion and slip regions. This condition is close to the adhesion optimum (maximum) for dry and wet curves and close to the minimum of the hydrodynamic lubrication for oil curves. 
- (c) $v_{x}=5.0 \%$ : most of the area is in slip. The creep curve is saturated for the static value of $\mathrm{COF}$.

Fig. 13 shows the tangential traction $\left(p_{x}\right)$, the slip $\left(\gamma_{x}\right)$ and the dissipated energy in each element $\left(T \gamma /\left.A\right|_{i}\right)$. For the same kinematic condition, depending on the friction parameters (dry, wet or oil) the tangential traction is different. With the presence of a lubricant the tangential traction is reduced while keeping the slip more or less constant. This produces a decrease in the energy dissipated. In (a), the dissipated energy for dry contacts is about four times greater than for wet and twenty times greater than oil. This difference is reduced when the creepage is increased. In (c), the dissipated energy for dry contacts is three times greater than for wet and seven times greater than oil. The absolute value for dry contacts reaches a maximum of approximately $11.7 \mathrm{~N} / \mathrm{mm}^{2}$ for (a), 18.6 for $(b)$ and 35.3 for $(c)$.

(a) $v_{x}=0.5 \%$
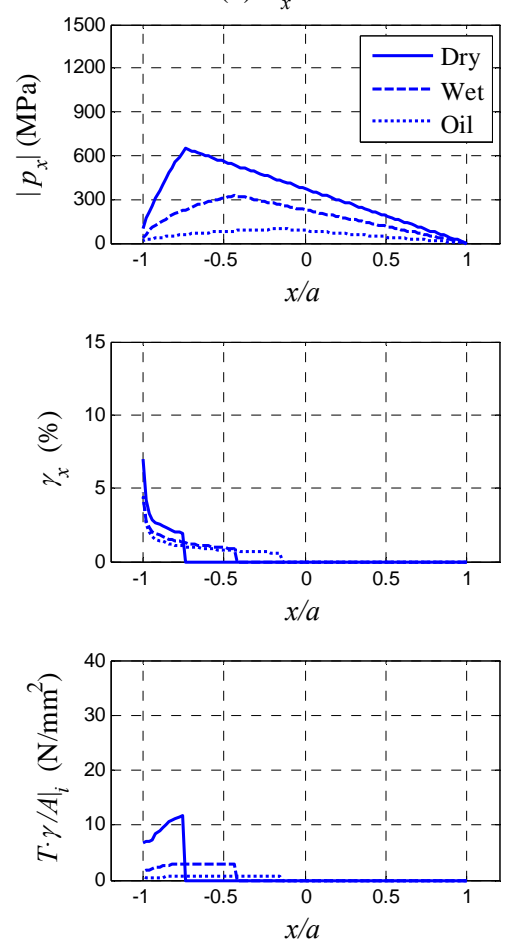

(b) $v_{x}=2.0 \%$
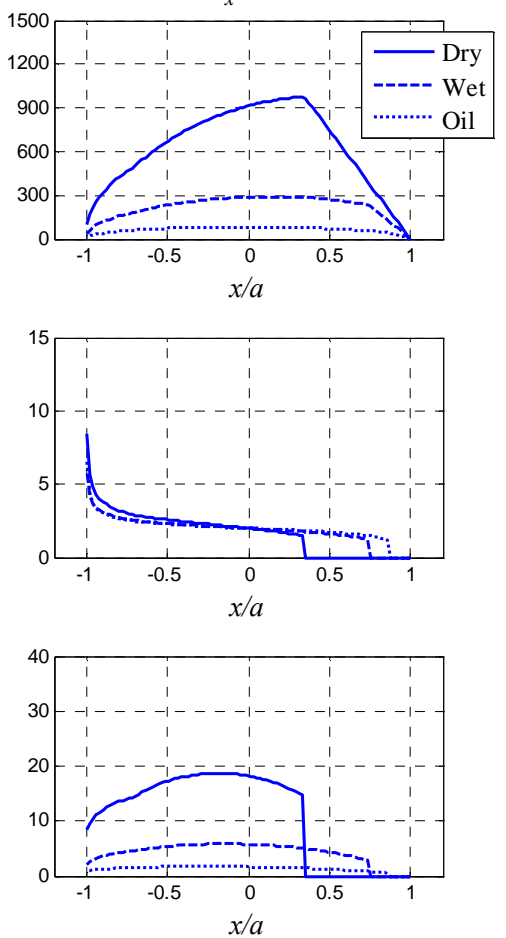

(c) $v_{x}=5.0 \%$
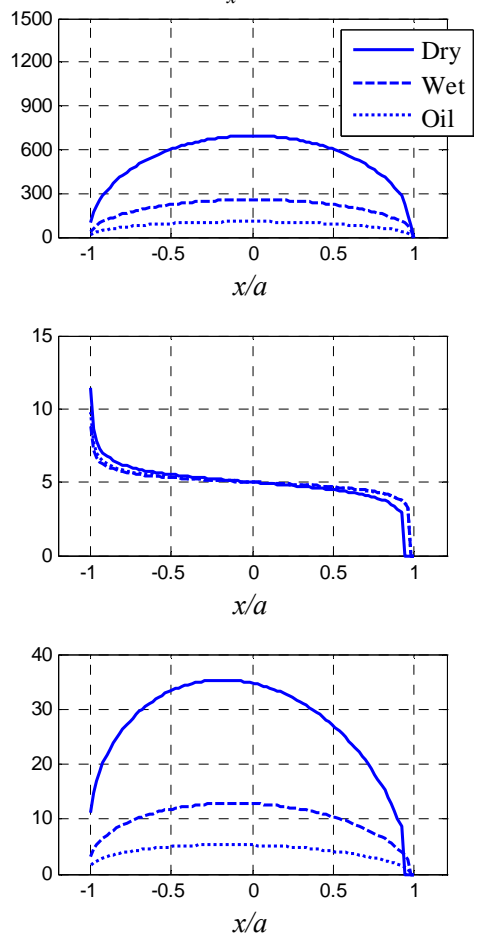

Fig. 13.Tangential tractions, slip and energy dissipated in the contact for dry, wet and oil conditions and for three different kinematic scenarios.

The global $T \gamma / A$ for each contact patch for dry,wet and oil conditions are calculated and plotted on the USFD wear law for dry contacts (Fig. 14). Firstly, the results for dry contacts are discussed. The points are located in the mild and severe regimes, as it is expected for tread wear in wheel-rail contact [29]. In (a), the global $T \gamma / A$ lies in the mild regime; although most of the area is in this regime some elements are in the severe regime. For a greater value of creepage $(b)$, the global $T \gamma / A$ moves to the severe regime. As seen in Fig. 13, the local distribution $T \gamma / A_{i}$ is greater than $10.4 \mathrm{~N} / \mathrm{mm}^{2}$ in most of the slip region. This change of regime is associated to the adhesion to slip transition. At this point, the slip area represents approximately $70 \%$ of the contact area. In the following case $(c)$, the contact is in the same regime, with a slip area that reaches $96 \%$ of the contact area. 
Secondly, the case of wet and oil contacts can be analysed. In those plots, the same wear law for dry contacts is supposed to be valid. Although this is generally assumed, it consists a very pessimistic scenario because with lubrication, the wear rate is greatly reduced [29], see Fig. 12-(b). With this assumption, all the contacts are in the mild regime except the wet contact for the scenario $(c)$, which is just in the transition from mild to severe regimes.

(a) $v_{x}=0,5 \%$

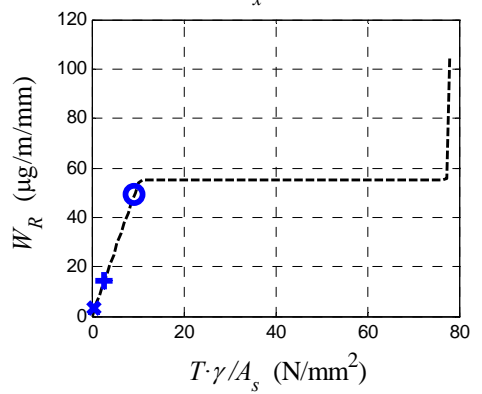

(b) $v_{x}=2,0 \%$

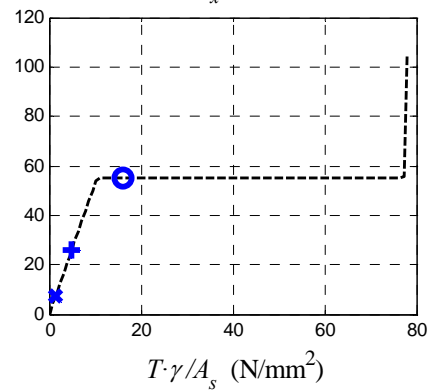

(c) $v_{x}=5,0 \%$

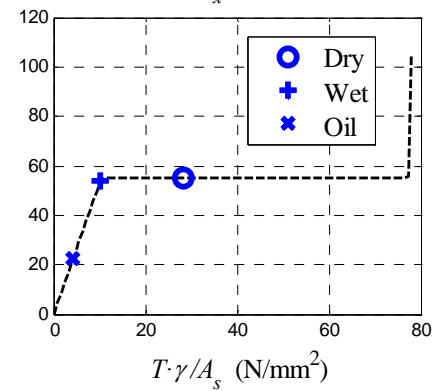

Fig. 14. Global wear index for the three contacts for dry, wet and oil. The points are located on the USFD wear law for dry contacts.

The wear estimation can be improved for wet contacts. A linear function with no offset can be fitted to the two points measured in Fig. 12-b). This is only an approximation for the wear obtained for wet contacts for small values of $T \gamma / A$ and is valid for a range $0<$ $T \gamma / A<14 \mathrm{~N} / \mathrm{mm}^{2}$ (see Table 10). Only mild regime is considered. Although it represents an improvement over the USFD for dry contacts, more laboratory testing would be required to get a better definition of the wear law.

Table 10. Wear law for R8T wheel and 900A rail materials for wet contacts.

\begin{tabular}{|c|c|c|}
\hline Regime & $T \gamma / A\left(\mathrm{~N} / \mathrm{mm}^{2}\right)$ & Wear Rate $\left(\mu \mathrm{g} / \mathrm{m} / \mathrm{mm}^{2}\right)$ \\
\hline $\mathrm{K}_{1}$ (mild $)$ & $T \gamma / A \leq 14.0$ & $0.09 T \gamma / A$ \\
\hline
\end{tabular}

Fig. 15 shows the dry and wet contacts plotted on their corresponding wear laws. Considering this new curve for wet contacts, in the three scenarios the wet contact lies in the mild regime, with a much smaller wear rate than the one predicted in Fig 14. As there is no wear law for contacts with oil, those contacts are not plotted.
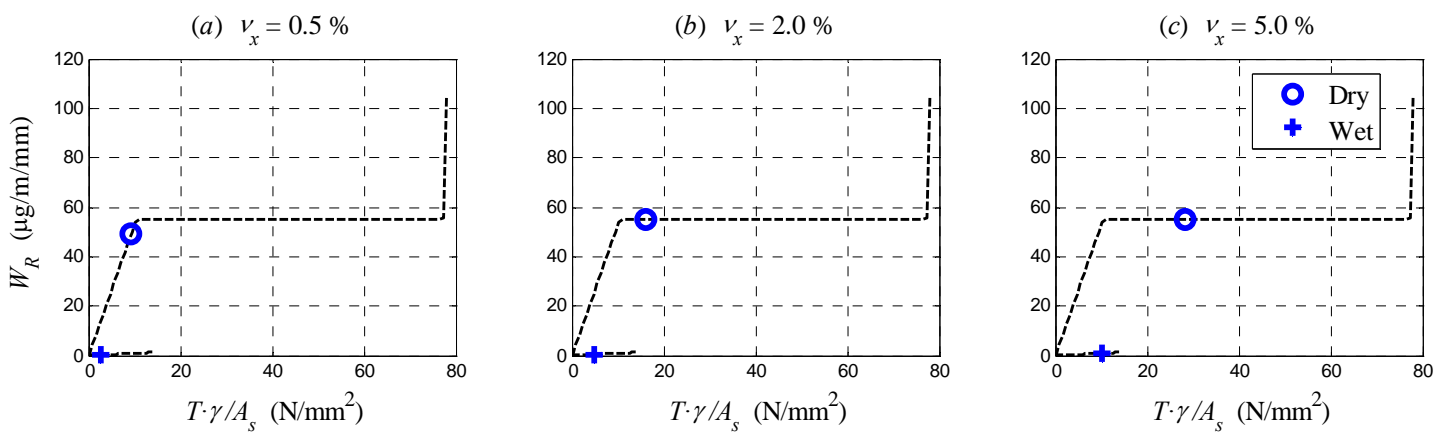

Fig. 15. Global wear index for dry and wet contacts, located on their corresponding wear law. 


\section{Application to wheel-rail contact}

In this section the Fastsim algorithm with variable coefficient of friction is adapted for the geometry of the wheel-rail contact. Due to the geometry of the surfaces in the contact, it is a three-dimensional problem and represents a further step than the twin disc case.

Based on the adapted model, two examples are presented in order to demonstrate the use of the formulations and parameters identified in this paper. These examples include the results of a wheelset on tangent track under high traction forces and the quasistatic behaviour of a vehicle traversing a curve. Magnitudes as pressure distribution within the contact patch, wheel-rail interaction forces and wear estimation are compared for both constant and variable coefficient of friction hypotheses.

\subsection{Modelling of the wheel-rail geometry}

The wheel-rail contact is analysed under the following hypotheses:

- The normal problem is solved using the equivalent ellipse method. The area of contact is obtained from the interpenetration of the wheel and rail profiles [32]. An equivalent ellipse is fitted to this contact patch [17] with semi-axes $a$ and $b$. The normal pressure distribution follows the Hertz model [22] (Fig. 16).

- For the tangential problem, the Fastsim algorithm with variable COF is used taking into account the flexibility coefficients $L_{1}, L_{2}$ and $L_{3}$ [3].

- The parameters which define the friction functions, identified for the longitudinal direction, are assumed to be valid for the lateral direction too. According to [11], this assumption can be accepted because those parameters influence mainly in the longitudinal direction where the maximum creep occurs.

- To calculate the $T \gamma / A$ corresponding to the elliptical area of contact, it is required to know the components of the slip vector. From equation (1), using $L_{1}$ for the equation for $x$ and $L_{2}$ for the equation for $y$ and calculating the derivative numerically (see the deduction of this formula in [33]), the expression for $\gamma_{x}$ and $\gamma_{y}$ is obtained. Dividing both formulas, it is possible to obtain the following relation which gives the ratio between the components of the slip vector:

$$
\frac{\gamma_{y}}{\gamma_{x}}=\frac{L_{2}}{L_{1}} \frac{p_{y}-p_{y}^{a d h}}{p_{x}-p_{x}^{a d h}}
$$

where the superindex $a d h$ is referred to the tangential stress under adhesion condition. Therefore, the angle of the slip vector $\alpha_{2}$ is

$$
\alpha_{2}=\tan ^{-1}\left(\frac{L_{2}}{L_{1}} \frac{p_{y}-p_{y}^{a d h}}{p_{x}-p_{x}^{a d h}}\right)
$$


and the $T \gamma / A$ index for the element $i, j$ of the contact patch can be calculated as

$$
\left.\frac{T \gamma}{A}\right|_{i, j}=\frac{T \gamma}{A}\left(x_{i}, y_{j}\right)=\mathbf{p}_{i, j} \cdot \gamma_{i, j}=p_{x_{i, j}} \cdot\left(\gamma_{i, j} \cos \left(\alpha_{2_{i, j}}\right)\right)+p_{y_{i, j}} \cdot\left(\gamma_{i, j} \sin \left(\alpha_{2_{i, j}}\right)\right)
$$

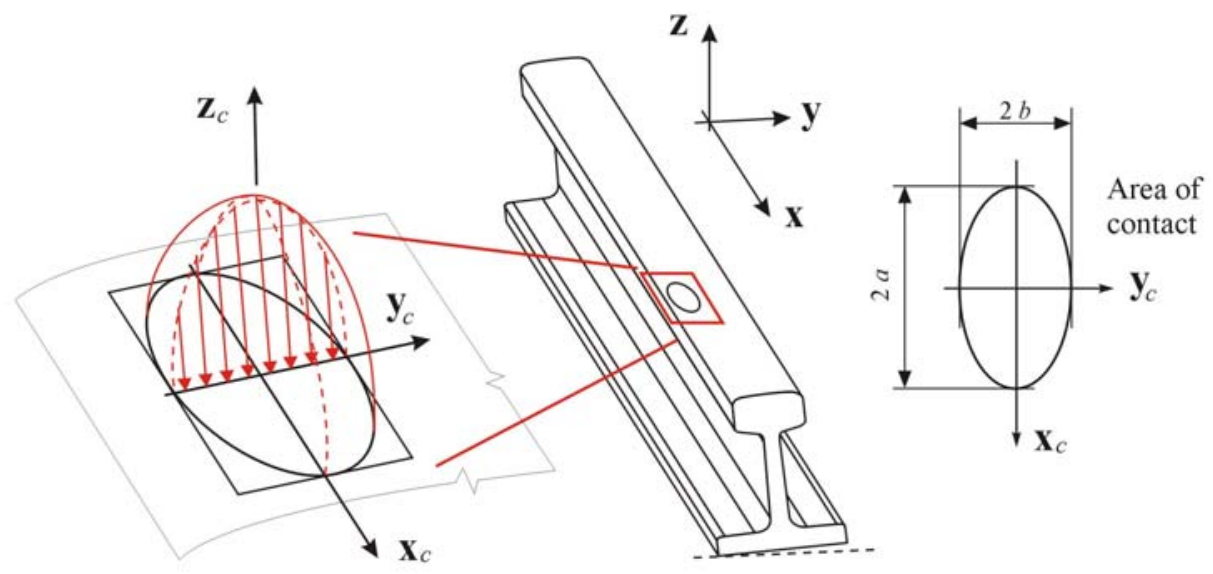

Fig. 16. Schematic representation of the wheel-rail geometry, with the elliptical area of contact of dimensions $2 a \cdot 2 b$ and the normal pressure distribution.

\subsection{Examples and numerical results}

The objective of this section is to compare the results obtained with the classic approach of constant COF with the approach of variable COF (which depends on the relative slip and on the pressure on the contact). On the one hand, the value of the constant COF is taken from the literature. On the other hand, for variable COF, the friction functions and the corresponding parameters identified from the experimental creep curves are used (Section 5).

Two examples are presented to show the implication of the consideration of the surface condition (presence/absence of a contaminant and its dependence on the pressure) on the wheel-rail interaction forces and wear.

Although the COF is a very important parameter to describe the tangential problem its value is not always offered in published papers. When given, the value of constant COF for dry steel-steel contacts is between 0.25 and 0.5 for applications of vehicle simulation or wear estimation. Some examples can be cited as Sinclair [34] with values between 0.24 and 0.4 for tread contacts, Shabana et al. [35] who use 0.5, Enblom et al. [36] with 0.3 , Tunna et al. [37] with 0.4, Simson et al. [38] test different values between 0.38 and 0.5, Matsumoto et al. [39] with 0.4 and Vuong et al. [40] takes 0.4. These values are stated in [41]. In this work a value of 0.4 for constant COF is taken with no slope reduction $(k=1)$. All these values represent the dry surface condition. Those values are not very different from the values identified for dry surfaces in Tables 3 and 6 (for pressures of $1500 \mathrm{MPa}$ and $900 \mathrm{MPa}$, respectively).

\subsubsection{Example 1: Tangent track under high traction forces}

In this first example, a centred wheelset on a tangent track is analysed under high traction forces (Fig. 17). The wheelset is travelling at a constant velocity with a 
prescribed value of longitudinal creepage (which simulates the traction force) and spin (to consider the conicity of the contact). The longitudinal creepage is taken close to the maximum of the creep curve for dry surfaces. The data used for the simulation is detailed in Table 11. The wheel and rail profiles are measured from new profiles. In this example, the relative position of the wheelset respect to the track is prescribed and does not depend on the wheel-rail forces. The objective of this example is to investigate the effect of contaminants on the tangential forces and on the wear estimation and the effect of the modelling with constant or variable COF.

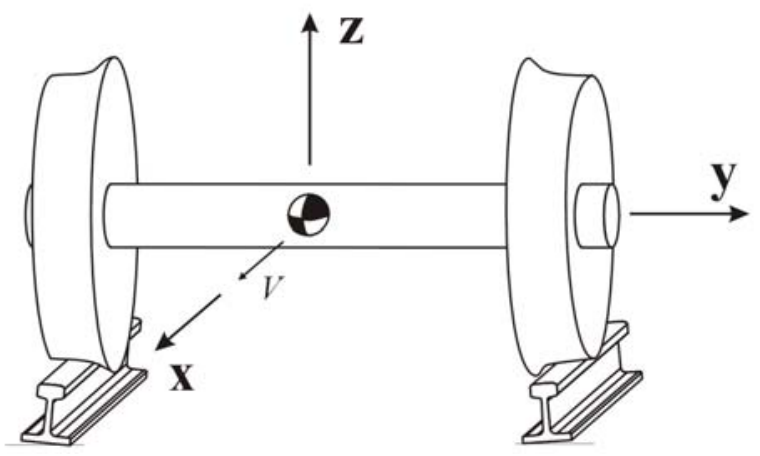

Fig. 17. Example 1: a wheelset centred on a tangent track.

Three different scenarios are considered: $(a)$ dry surfaces, $(b)$ wet surfaces and $(c)$ tangential problem modelled for constant COF $\mu=0.4$. (a) and (b) consider a variable COF with the friction parameters and slope reduction identified in Section 5.

Table 11. Data used for the simulation.

\begin{tabular}{|l|r|}
\hline Velocity & $90 \mathrm{~km} / \mathrm{h}$ \\
\hline Gauge & $1435 \mathrm{~mm}$ \\
\hline Radius of the wheel & $450 \mathrm{~mm}$ \\
\hline Distance between contact points & $1500 \mathrm{~mm}$ \\
\hline Wheel profile & S1002 \\
\hline Rail profile & UIC60 \\
\hline Rail slant & $1: 20$ \\
\hline Wheelset mass & $1500 \mathrm{~kg}$ \\
\hline Weight per axle & $120 \mathrm{kN}$ \\
\hline Longitudinal creepage $v_{x}$ & $1.2 \%$ \\
\hline Lateral creepage $v_{y}$ & $0.0 \%$ \\
\hline Spin $\varphi(1 / \mathrm{m})$ & $6.55 \%$ \\
\hline
\end{tabular}

As a result of the normal problem, there are two areas of contact between the wheelset and the rail, one for each wheel and symmetric (Fig. 18). The dimensions of the areas of contact and the maximum pressure are stated in Table 12. The maximum Hertzian pressure is used to interpolate the friction parameters and slope reduction as explained in section 5.2. 

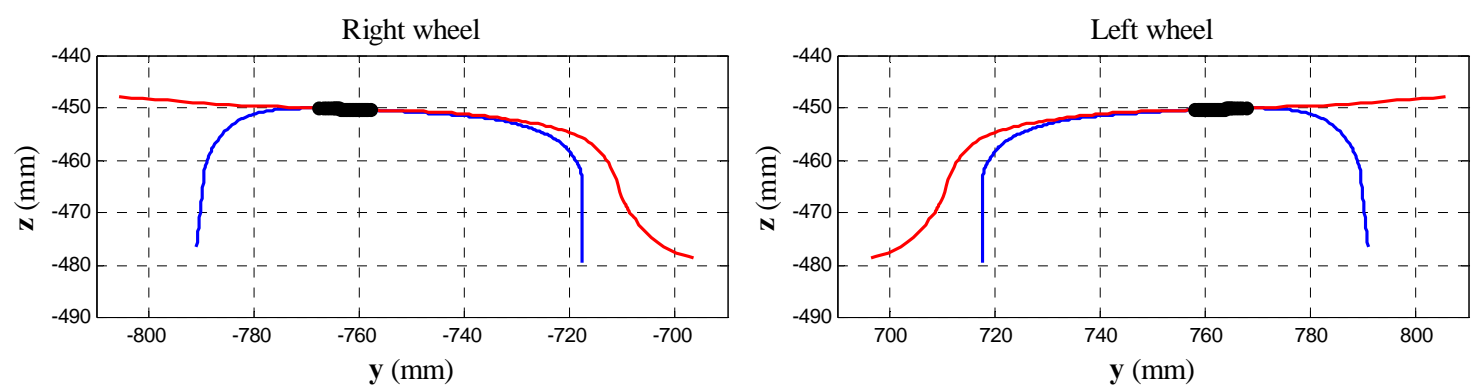

Fig. 18. Geometry of the contact and contact areas for Example 1.

Table 12. Results of the normal problem.

\begin{tabular}{|c|r|}
\hline$p_{z_{o}}$ & $1121.14 \mathrm{MPa}$ \\
\hline$a$ & $5.95 \mathrm{~mm}$ \\
\hline$b$ & $4.26 \mathrm{~mm}$ \\
\hline
\end{tabular}

Once solved the normal problem, the creep curves are calculated for the three scenarios (Fig 19) where the longitudinal creepage is in the range 0-6\%. For dry and wet conditions, using the value of the maximum Hertzian pressure $\left(p_{z_{o}}\right)$ of Table 12, the values of the friction parameters and slope reduction are interpolated as explained in Section 5.2 (between the values identified for the two levels of pressure, $900 \mathrm{MPa}$ and $1500 \mathrm{MPa}$ ). Then, the creep curves for dry and wet are calculated applying Fastsim with variable COF and slope reduction; on the other hand, the creep curve for $\mu=0.4$ is calculated applying Fastsim (with no slope reduction and no pressure dependence). The differences in the three curves illustrate the differences among the three scenarios. The dot in the curves indicates the position under study.

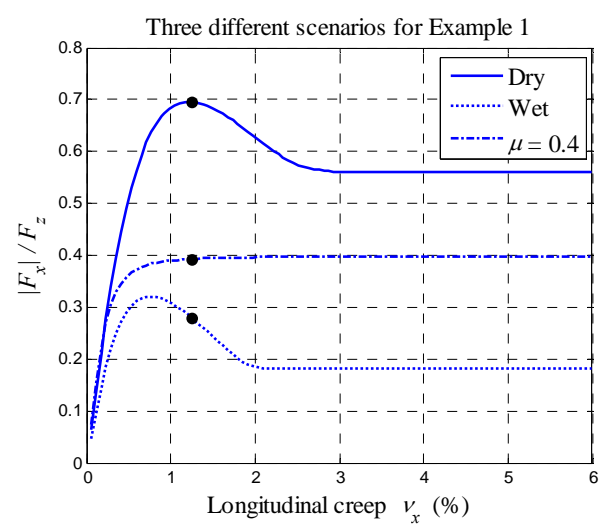

Fig. 19. Creep curves corresponding to the three scenarios. The black point represents the kinematic condition studied.

To evaluate the three scenarios, in Fig. 20 the longitudinal stress distribution and the energy dissipation distribution $\left(T \gamma /\left.A\right|_{i}\right)$ within the area of contact are plotted. In the condition studied in this example, close to the adhesion optimum for dry surfaces (see Fig. 19), the longitudinal stress is much grater than the lateral stress created by the spin. In order to get a clear shape of the tangential stress distribution, only the longitudinal component is represented. Nevertheless, the effect of spin is considered in the calculation of the energy dissipation $\left(T \gamma /\left.A\right|_{i}\right)$ in Fig. 20, through the lateral stress in equation (18). 
In both magnitudes the adhesion region is easy to distinguish (where the pressure distribution is linear and the $T \gamma /\left.A\right|_{i}$ is zero). Integrating those magnitudes, the total force and the global energy dissipated $(T \gamma / A)$ can be obtained (Table 13).
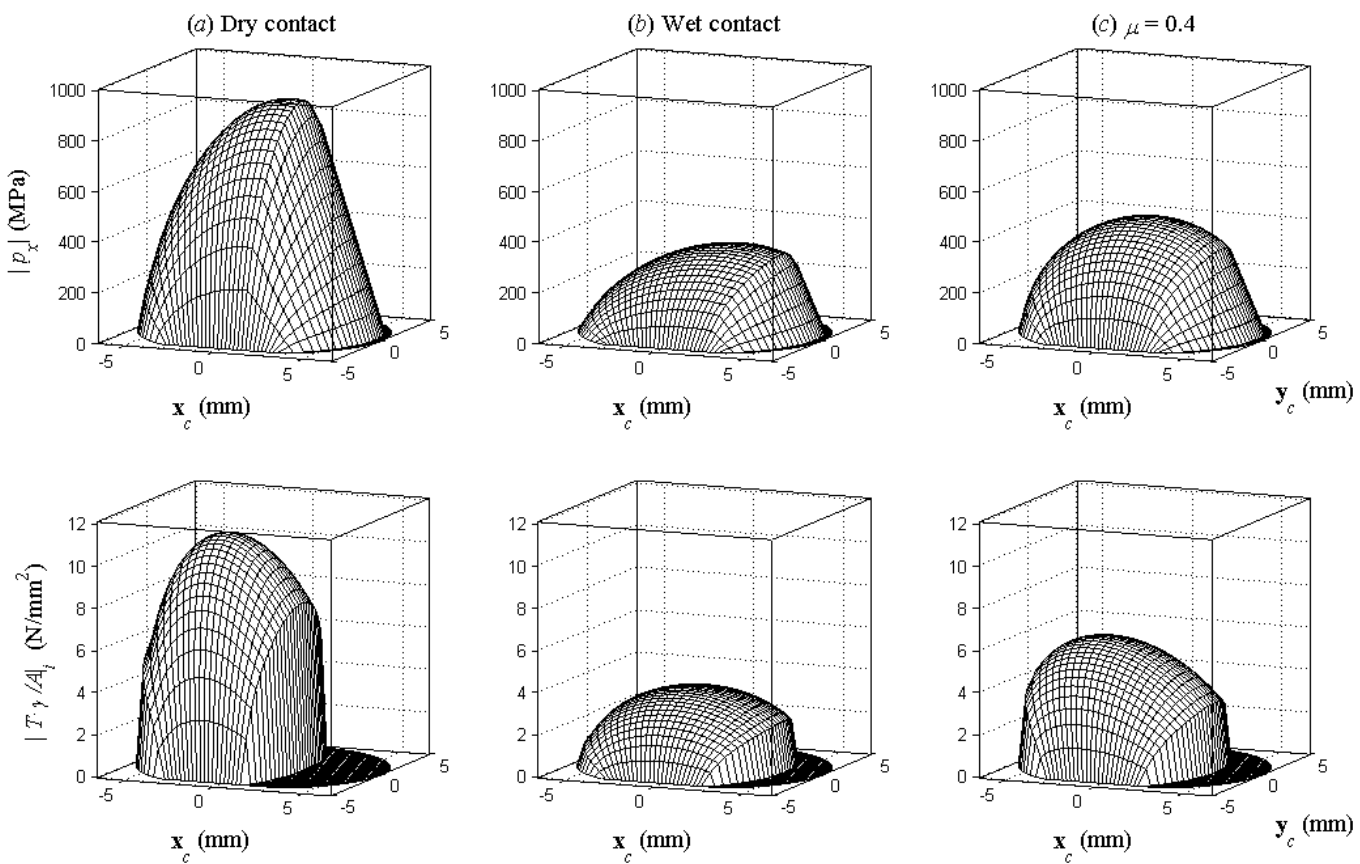

Fig. 20. Pressure distribution $\left(p_{x}\right)$ and $T \gamma / A$ distribution on the contact area under the three scenarios.

Table 13. Results of the tangential problem.

\begin{tabular}{|c|c|c|c|}
\cline { 2 - 4 } \multicolumn{1}{c|}{} & Dry & Wet & $\mu=0.4$ \\
\hline$F_{x}(\mathrm{kN})$ & 41.42 & 17.00 & 23.24 \\
\hline$T \gamma / A$ & 7.90 & 2.56 & 3.99 \\
\hline
\end{tabular}

Fig. 21 plots on the USFD wear law the global energy dissipated in the three contacts studied. $(a)$ and $(c)$ are plotted on the wear law for dry contacts. To illustrate the effect of the consideration of a different wear law, the contact $(b)$ is plotted on the wear law for dry contacts (Table 9) and on the wear law for wet ones (Table 10). In both cases the contact is in the mild regime but the associated wear rate greatly changes. In this case the effect of the contaminant is taken into account in the computation of the $T \gamma / A$ which represents the position on the $\mathrm{x}$-axis and in the wear law considered, which represents the position in the $y$-axis. 

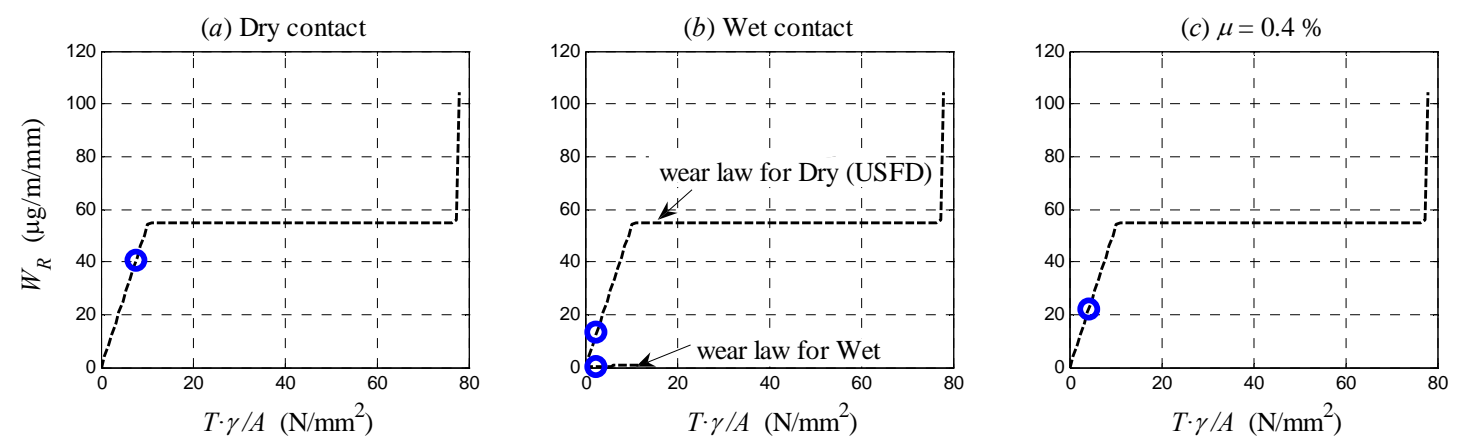

Fig. 21. Global energy dissipated in the contact.

The comparison of $(a)$ with $(c)$ gives an idea of the error made in the contact modelling of the system. For the considered kinematics, the error assuming a constant COF $\mu=0.4$ when the surfaces are actually dry is about $45 \%$ in forces and in energy dissipated (taking the dry contact as a reference).

The comparison of $(a)$ with $(b)$ represents the error made assuming a different surface contamination. If the surfaces were wet, but assumed dry, the error reach $144 \%$ in forces and $200 \%$ in energy dissipated (taking the wet contact as a reference). Furthermore, if the estimated wear law for wet contacts is used, the estimated error in wear can reach values up to $250 \%$.

The error in forces would affect the motion of the wheelset over the track in a dynamic simulation (and, therefore, the accumulated damage on the wheel/rail profiles).

\subsubsection{Example 2: Vehicle traversing a curve}

In this second example the quasistatic behaviour of a full vehicle traversing a curve is studied. This case is a special case in the overall study of the behaviour of a rail vehicle. The purpose of this kind of analysis is to predict the steady-state attitude and the resulting wheel-rail and suspension forces [41]. The quasistatic position is an equilibrium position of the bogies of the vehicle when traversing a curve. The objective of this example is to investigate the effect of the constant/variable COF on the wheel/rail interaction forces and equilibrium position of the wheelsets in the track.

The surfaces in the contact are assumed to be dry. Two different contact models are tested: $(a)$ a model of variable COF and $(b)$ a model for constant COF. Firstly, the method for variable COF and dry surfaces takes the friction parameters and slope reduction identified from experimental data detailed in Section 5; those parameters are dependent on the pressure. Secondly, for constant COF, a constant value of $\mu=0.4$ and no slope reduction are chosen.

Table 14. Data used for the simulation (example 2).

\begin{tabular}{|l|r|}
\hline Velocity & $90 \mathrm{~km} / \mathrm{h}$ \\
\hline Radius of the curve & $2000 \mathrm{~m}$ \\
\hline Cant & $100 \mathrm{~mm}$ \\
\hline Non-balanced acceleration & $0.31 \mathrm{~m} / \mathrm{s}^{2}$ \\
\hline Bogie wheelbase & $2560 \mathrm{~mm}$ \\
\hline Bogie mass & $3300 \mathrm{~kg}$ \\
\hline Carbody mass & $36000 \mathrm{~kg}$ \\
\hline Stiffness of primary susp. $\left(k_{x}\right)$ & $32 \mathrm{MN} / \mathrm{m}$ \\
\hline
\end{tabular}


For the calculation, the same data from the previous example is used along with data from Table 14. The vehicle is composed of two bogies of type B (two wheelsets), and it negotiates a canted curve of constant radius with cant deficiency. A typical set of four coordinates is used to model the motion of the wheelset in the track [42], which are: lateral displacement $y$, vertical displacement $z$, roll angle $\phi$ and yaw angle $\psi$. Fig. 22 shows a schematic representation of the wheelsets of the front bogie and its attitude, showing the coordinates $y$ and $\psi$. Only the results for the wheelsets of the leading bogie are presented.

Table 15 and Fig. 23 show the results of the quasistatic problem for the wheelsets of the leading bogie under the two hypotheses. For both cases, the leading wheelset moves outside the curve and develops a positive angle of attack $(\psi>0)$ whereas the trailing wheelset stays in a position close to the centre of the track and develops a negative angle of attack. This is the expected behaviour for the leading bogie. However, the differences between the wheelsets attitudes for the two cases studied are noticeable. As the friction is greater for $(a)$, the leading wheelset has a smaller lateral displacement $y$ than $(b)$, which is near flanging. This same behaviour is experienced by the angle of attack $\psi$. The trailing wheelset moves inside the curve for $(a)$, and stays in the centred position for $(b)$; in both scenarios the angle of attack is negative.

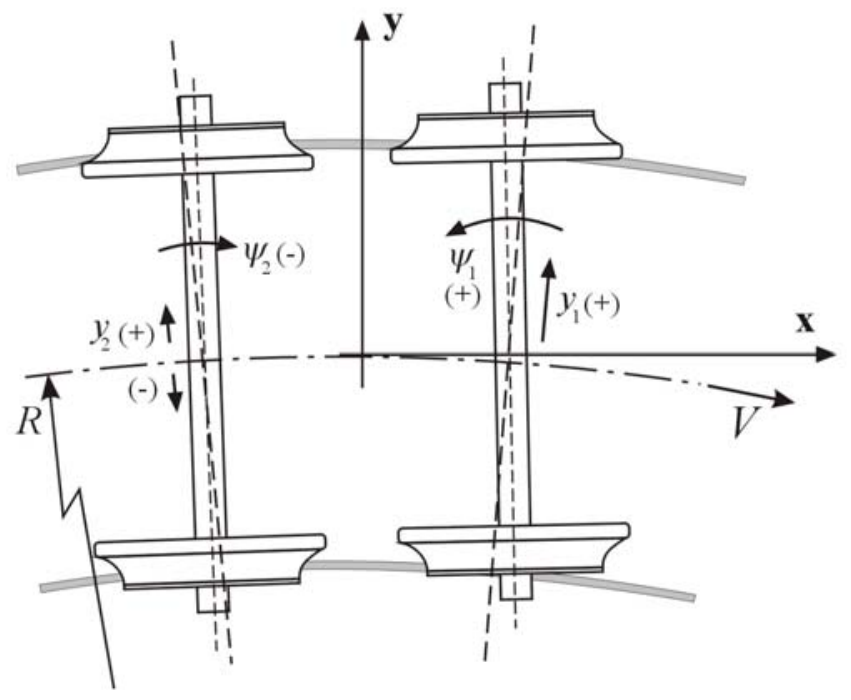

Fig. 22. Example 2: a vehicle traversing a curve (wheelsets of the leading bogie).

Table 15. Wheelsets attitudes.

\begin{tabular}{|c|c|c|c|}
\cline { 2 - 4 } \multicolumn{1}{c|}{} & $(a)$ Dry surfaces & $(b) \mu=0.4$ & $\% \Delta$ respect dry \\
\hline Leading & $y_{1}=4.73 \mathrm{~mm}$ & $y_{1}=5.82 \mathrm{~mm}$ & $23 \%$ \\
\cline { 2 - 4 } wheelset & $\psi_{1}=0.26 \mathrm{mrad}$ & $\psi_{1}=0.52 \mathrm{mrad}$ & $100 \%$ \\
\hline Trailing & $y_{2}=-2.39 \mathrm{~mm}$ & $y_{2}=0.04 \mathrm{~mm}$ & $100 \%$ \\
\cline { 2 - 4 } wheelset & $\psi_{2}=-0.59 \mathrm{mrad}$ & $\psi_{2}=-0.44 \mathrm{mrad}$ & $25 \%$ \\
\hline
\end{tabular}

The result of this simulation could describe a generic case of a railroad vehicle traversing a curve. Fig. 23 represents the contact patches generated in the wheel-rail contact for the wheelsets of the leading bogie. Their main characteristics are summarized in Table 16. The number and shape of contact patches depend on the relative position of the wheelset in the track and it is very sensitive to the wheel-rail relative position. 
For the parameters used in the calculation, in this case the leading wheelset for $(a)$ develops three contact patches (two on the outer side and one on the inner side) whereas for $(b)$, there are only two (one at each side). This configuration is the result of the relative position of the wheelset respect to the track, which is the solution of the equilibrium position of the solids of the vehicle to the applied external forces (kinematics and wheel-rail contact forces).

The wheelset attitude in $(a)$ is a situation where the load is being transferred from one contact patch to the other. In $(b)$, where the lateral displacement is greater, the load has completely been transferred to the contact patch near the wheel flange fillet. This different contact configuration will affect the wear estimation.

For the trailing wheelset the configuration is very close to a centred wheelset. Even though the lateral displacement is different for $(a)$ and $(b)$, the configuration of the contact is very close.
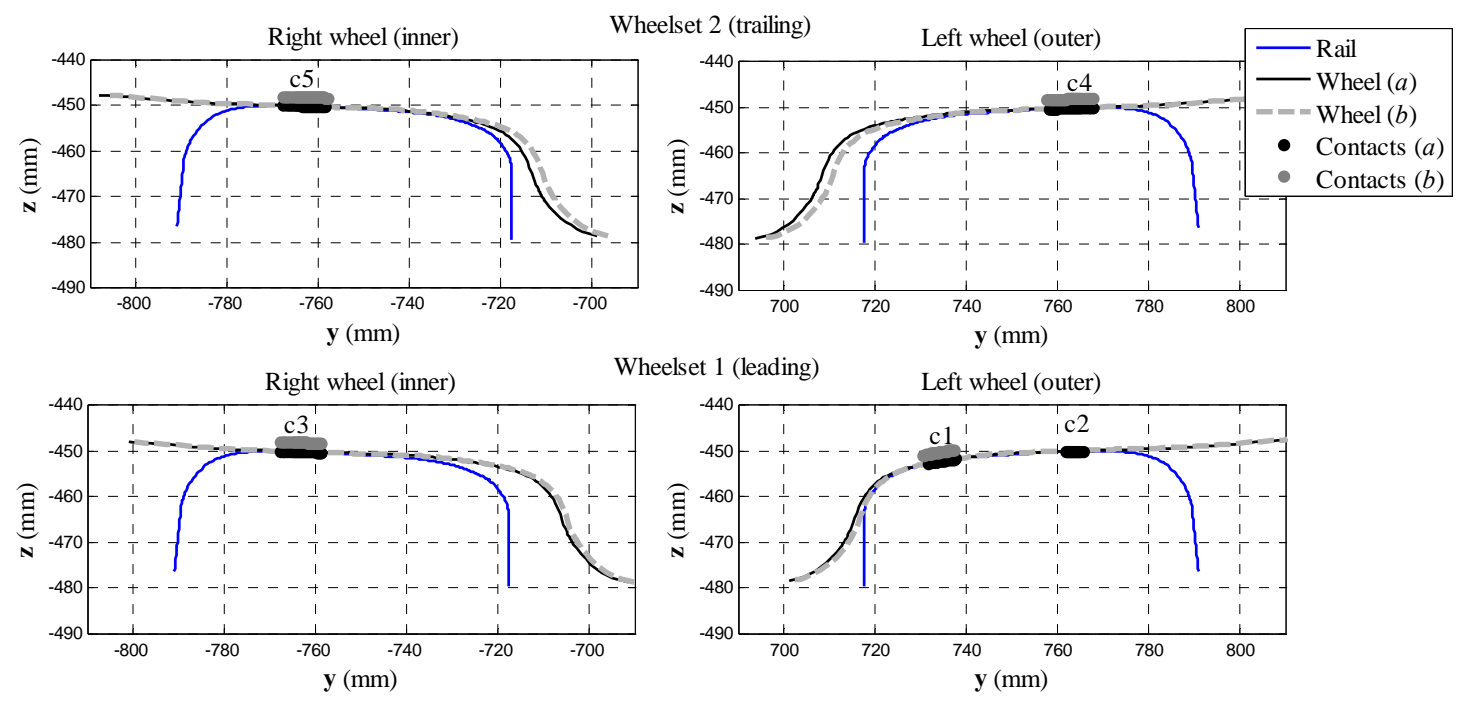

Fig. 23. Schematic representation of the attitude of the two wheelsets of the leading bogie of the vehicle. The two quasistatic positions and contact patches are plotted together for $(a)$ variable COF and $(b)$ constant COF.

Table 16. Summary of the results of the contact (*a null value of $T \gamma / A$ means that the whole contact patch is in adhesion).

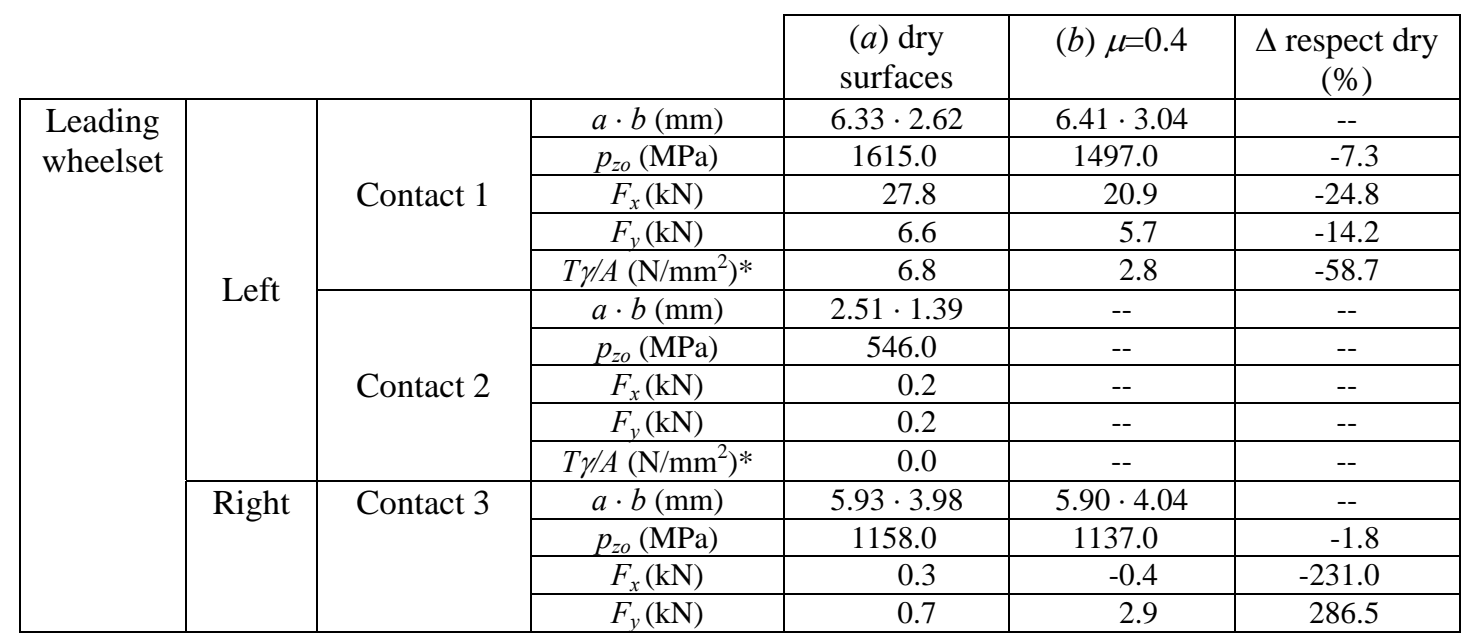




\begin{tabular}{|c|c|c|c|c|c|c|}
\hline & & & $T \gamma / A\left(\mathrm{~N} / \mathrm{mm}^{2}\right)^{*}$ & 0.0 & 1.3 & -- \\
\hline \multirow{10}{*}{$\begin{array}{c}\text { Trailing } \\
\text { wheelset }\end{array}$} & \multirow{5}{*}{ Left } & \multirow{5}{*}{ Contact 4} & $a \cdot b(\mathrm{~mm})$ & $6.07 \cdot 4.24$ & $6.00 \cdot 4.49$ & -- \\
\hline & & & $p_{z o}(\mathrm{MPa})$ & 1160.0 & 1101.00 & -5.1 \\
\hline & & & $F_{x}(\mathrm{kN})$ & -4.5 & -3.6 & -19.4 \\
\hline & & & $F_{y}(\mathrm{kN})$ & -3.2 & -2.3 & -26.7 \\
\hline & & & $T \gamma / A\left(\mathrm{~N} / \mathrm{mm}^{2}\right)^{*}$ & 3.4 & 1.2 & -63.0 \\
\hline & \multirow{5}{*}{ Right } & \multirow{5}{*}{ Contact 5} & $a \cdot b(\mathrm{~mm})$ & $5.84 \cdot 4.02$ & $5.77 \cdot 4.33$ & -- \\
\hline & & & $p_{z o}(\mathrm{MPa})$ & 1123.00 & 1059.0 & -5.7 \\
\hline & & & $F_{x}(\mathrm{kN})$ & 5.0 & 3.3 & -32.3 \\
\hline & & & $F_{y}(\mathrm{kN})$ & -4.8 & -4.6 & -4.8 \\
\hline & & & $T \gamma / A\left(\mathrm{~N} / \mathrm{mm}^{2}\right)^{*}$ & 3.3 & 1.1 & -66.3 \\
\hline
\end{tabular}
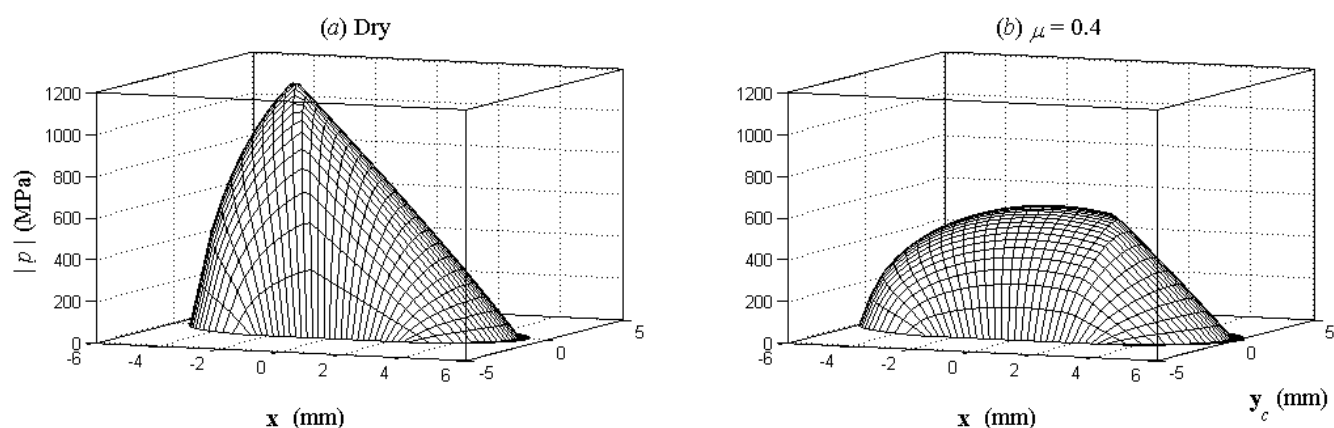

$\mathbf{x}(\mathrm{mm})$

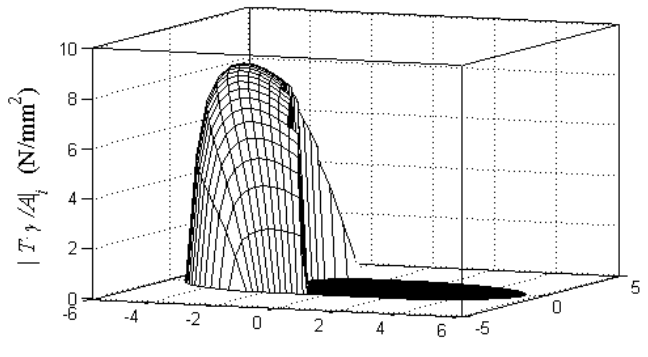

$\mathbf{x}(\mathrm{mm})$

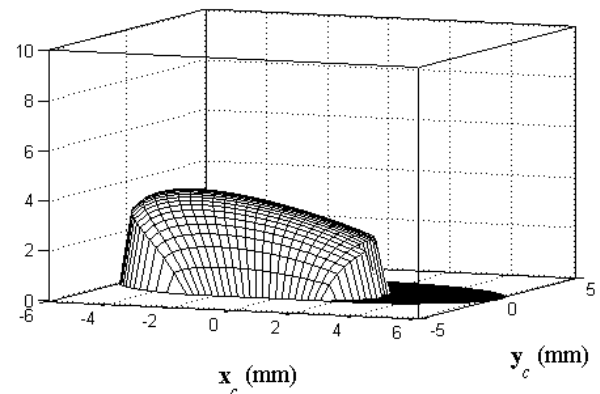

Fig. 24. Tangential pressure and the local $T \gamma / A$ distributions on the contact 1.

Finally, contact 1 obtained for the two hypotheses is compared. Fig 24 represents the tangential stress distribution and the energy dissipated in the contact patch as a wear indicator. Although the maximum normal pressure is very close for both cases, the maximum tangential pressure is $24 \%$ smaller for case $(b)$. This is because the COF is less for $(b)$ than for $(a)$. Furthermore, the energy dissipated is different for both patches. The wear estimation offered by the constant COF is $58 \%$ less than variable the COF. In both scenarios, the whole contact patch is in the mild regime.

The results of this example show that the consideration of different friction functions to model the tangential problem leads to different motion of the wheelsets on the track (although they follow the same trend) and, especially, different wear patterns on the wheel and rail profiles.

\section{Discussion}

In this work, numerical methods to model the tangential problem with variable friction have been studied. An improvement has been added to fit experimental friction data, which is the inclusion of the reduction of the initial slope. Different friction functions have been tested and the friction parameters that define those functions have been identified from experimental measurements. 
When studying a contact subjected to shear forces there is a lot of scatter, especially in the presence of contaminants. However, the experimental creep curves taken from published references seem consistent compared with previous work and reproduce the expected behaviour for variable friction. Therefore, the parameters identified from these curves are expected to offer a better simulation than a simple estimation or just the consideration of a single value for the coefficient of friction. In this line, more experimental measurements are required to improve the accuracy in the estimation of the friction parameters, covering different surface contaminants and contact conditions (pressure, rolling velocity, room temperature).

Different friction functions have been tested: two friction functions depending on three parameters (exponential function $\mathrm{f}_{1 \mathrm{a}}$ and piecewise function $\mathrm{f}_{1 \mathrm{~b}}$ ) and a friction function depending on four parameters $f_{2}$. When possible, the function with a smaller number of parameters has been used: i.e in the case of dry contacts, a friction function with three parameters was enough, but for the case of oil, a friction function depending on an extra parameter was required. This was done for the sake of efficiency.

Fig. 25 shows the relative computation time for the different friction functions; it corresponds to the computation time of a Fastsim run (with 30x30 divisions of the area of contact), using the friction functions considered in this paper. The differences are due to the procedure required to solve the system of non-linear equations. The computation time for $\mathrm{f}_{2}$ is much greater than for any function $\mathrm{f}_{1}$. Comparing $\mathrm{f}_{1 \mathrm{a}}$ and $\mathrm{f}_{1 \mathrm{~b}}$, in Section 5 it has been shown than both functions produce very similar results and can be used in the same conditions. However $\mathrm{f}_{1 \mathrm{~b}}$ involves a much lower computational cost because it is a linear approximation of a non-linear function. Therefore, when three friction parameters are required it is advisable to use $\mathrm{f}_{1 \mathrm{~b}}$. With this function, the computational cost of variable coefficient of friction compared to constant coefficient of friction is only 1.7 times greater, whereas it is 10 times greater for $f_{1 a}$ and 20 times greater for $f_{2}$. The function $\mathrm{f}_{2}$ when used to model lubricated contacts reproduces the Stribeck curve.

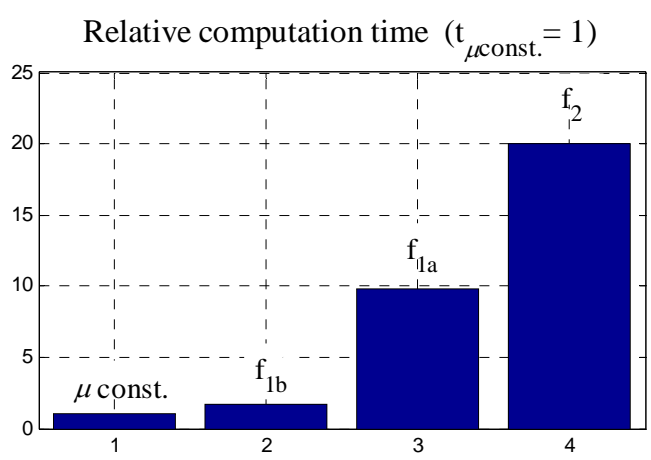

Fig. 25. Relative computation time with the different algorithms, taking $\mathrm{t}_{\mu \mathrm{const}}=1$.

In the case of dry surfaces or in the presence of water or oil in the interface, the effect of the pressure on the friction parameters and slope reduction has been investigated. Although the measurements are consistent, possibly more experimental work is required to establish a clear relation between the pressure and the shear behaviour. Nevertheless, the consideration of the dependence of the friction on the pressure does not involve an increase in computational cost and the results presented in this work can be used as a first approximation. 
For the twin disc geometry, the implication of the surface contamination on wear has also been shown. The available USFD wear law is only valid for dry contacts, so for the presence of a surface contaminant, a new wear law is needed. Furthermore, the consideration of a different contaminant changes the value of the COF which gives new estimation of the tangential forces and slip. As an example, lubrication greatly reduces the energy dissipation in the contact patch. Furthermore, the use of USFD for dry contacts would overestimate the wear rate for the presence of oil.

Another aspect to highlight is the adaptation from the geometry of the twin disc (which is a two-dimensional problem) to the geometry of the wheel rail contact (which is a full 3D problem). Special care has been taken in order to ensure that the values extracted from the experimental measurements can be applied to the wheel-rail contact.

Finally, two examples have been presented for the wheel-rail geometry. The results show the differences between the classic approach using constant COF and the modelling of variable COF. Both approaches offer different estimation in the interaction forces and wear.

As a future work, this algorithm of Fastsim with variable coefficient of friction and slope reduction must be tested in the field of dynamic simulation. Constant and variable friction approaches can be compared. For the case of variable friction, different conditions of the wheel-rail interface can be modelled such as dry surfaces, moisture on the wheel tread and the presence of oil in a flange contact. On the experimental field, more testing is required to evaluate the effect of the contaminants on the wear rate.

\section{Conclusions}

- Different tangential contact models considering Fastsim with variable COF have been studied. An improvement in the slope reduction for small creepages has been considered to be able to reproduce experimental measurements.

- Different friction functions have been used to model the local behaviour of a point in the contact. A new friction function, with resembles the Stribeck curve, has been defined to model increasing creep curves after saturation. Depending on the shape of the creep curve, an adequate friction function, using the minimum number of parameters, has been chosen. Their application and their computational cost have been presented.

- A new procedure has been presented to extract the friction parameters and slope reduction from twin disc experimental measurements.

- The parameters extracted from the twin disc geometry for a steel-steel contact with a surface condition (presence/absence of a contaminant and its dependence on the pressure) have been adapted to be used with the wheel-rail geometry, with the same steel-steel contact and surface condition.

- The effect of the contaminants on wear estimation is analysed. The available wear laws are valid for dry conditions in the interface. More experimental work is required to define suitable wear laws for non-dry contacts. 
- Two examples have been presented to compare the solution between the classic approach (constant friction) and a variable friction approach. The difference can reach values greater than $200 \%$ in contact forces and $60 \%$ in wear estimation for dry contacts.

\section{Acknowledgments}

This research was supported by Universitat Politecnica de Valencia (Spain).

\section{References}

[1] J. Evans, M. Berg, Challenges in simulation of rail vehicle dynamics. Vehicle System Dynamics 47 (2009) 1023-1048.

[2] J.G. Giménez, A. Alonso, E. Gomez, Introduction of a friction coefficient dependent on the slip in the FastSim algorithm. Vehicle System Dynamics 43 (2005) 233-244.

[3] J.J. Kalker, A fast algorithm for the simplified theory of rolling contact. Vehicle System Dynamics 11 (1982) 1-13.

[4] T. Ohyama, Tribological studies on adhesion phenomena between wheel and rail at high speeds. Wear 144 (1991) 263-275

[5] E. Magel, Y. Liu, Study of friction - Measurement analysis and practical implications for the wheel/rail contact. 8th International Conference on Contact Mechanics and Wear of Rail/Wheel Systems, Firenze, Italy, September 15-18, 2009.

[6] M. Ertz, F. Bucher, Improved creep force model for wheel/rail contact considering roughness and temperature, Vehicle System Dynamics. Supplement 37 (2002) 314-325.

[7] Z.Y. Shen, J.K. Hedrick, J.A. Elkins, A comparison of alternative creep force models for rail vehicle dynamics analysis. Proceedings of the $8^{\text {th }}$ IAVSD Symposium held at MIT, Cambridge (MA), Aug 1983. 591-605.

[8] K. Knothe, A. Theiler, Normal and tangential contact problem with rough surfaces. $2^{\text {nd }}$ MiniConference on Contact Mechanics and Wear of Rail/Wheel Systems, Budapest, July 29-31, 1996.

[9] F. Bucher, K. Knothe, A. Theiler, Normal and tangential contact problem of surfaces with measured roughness, Wear 253 (2002) 204-218.

[10] O. Polach, Influence of locomotive tractive effort on the forces between wheel and rail. Vehicle System Dynamics 25 (2001) 7-22.

[11] O. Polach, Creep forces in simulations of traction vehicles running on adhesion limit. Wear 258 (2005) 992-1000.

[12] J.B. Nielsen, A. Theiler, Tangential contact problem with friction coefficients depending on sliding velocity. Proceedings of Second Mini-Conference on Contact Mechanics and Wear of Rail/Wheel Systems. Budapest (1996) 44-51.

[13] J. Piotrowski, Kalker's algorithm fastsim solves tangential contact problems with slip-dependent friction and friction anisotropy. Vehicle System Dynamics 48 (2010) 869-889.

[14] D.T. Eadie, M. Santoro, J. Kalousek, Railway noise and the effect of top of rail liquid friction modifiers: changes in sound and vibration spectral distributions in curves. Wear 258 (2005) 11481155.

[15] A.D. Monk-Steel, D.J. Thompson, F.G. De Beer, M.H.A. Janssens, An investigation into the influence of longitudinal creepage on railway squeal noise due to lateral creepage. Journal of Sound and Vibration 293 (2006) 766-776.

[16] G. Xie, P.D. Allen, S.D. Iwnicki, A. Alonso, D.J. Thompson, C.J.C. Jones, Z.Y. Huang, Introduction of falling friction coefficients into curving calculations for studying curve squeal noise. Vehicle System Dynamics 44 (2006) 261-271.

[17] A. Rovira, A. Roda, M.B. Marshall, H. Brunskill, R. Lewis, Experimental and numerical modelling of wheel-rail contact and wear, Wear 271 (2011) 911-924

[18] J. Williams, Engineering Tribology, Cambridge University Press, Cambridge, 2005. 
[19] J.J. Kalker, Survery of wheel-rail rolling contact theory. Vehicle System Dynamics 5 (1979) 317358.

[20] D.I. Fletcher, J.H. Beynon, Development of a machine for closely controlled rolling contact fatigue and wear testing. Journal of Testing and Evaluation 28 (2000) 267-275.

[21] E.A. Gallardo-Hernandez, R. Lewis, Twin disc assessment of wheel/rail adhesion. Wear 265 (2008) 1309-1316.

[22] K.L. Johnson, Contact Mechanics. Cambridge University Press, Cambridge, 1987.

[23] J.J. Kalker, Three-Dimensional Elastic Bodies in Rolling Contact, Kluwer Academic Publishers, Dortercht/Boston/London, 1990.

[24] R. Lewis, E.A. Gallardo-Hernandez, T. Hilton, T. Armitage, Effect of oil and water mixtures on adhesion in the wheel/rail contact. Proc. IMechE part F: Journal of Rail Rapid Transit 223 (2009) 275-283.

[25] O. Arias-Cuevas, Z. Li, R. Lewis, E.A. Gallardo-Hernandez, Rolling-sliding laboratory tests of friction modifiers in dry and wet wheel-rail contacts. Wear 268 (2010) 543-551.

[26] F. Bucher, A.I. Dmitriev, M. Ertz, K. Knothe, V.L. Popov, S.G. Psakhie, E.V. Shilko, Multiscale simulation of dry friction in wheel/rail contact. Wear 261 (2006) 874-884.

[27] D.T. Eadie, J. Kalousec, K.C. Chiddick, The role of high positive friction (HPF) modifier in the control of short pitch corrugations and related phenomena. Wear 253 (2002) 185-192.

[28] D.T. Eadie, J. Kalousek. Railway Age (June 2001) 48-50.

[29] R. Lewis, R.S. Dwyer-Joyce, Wear mechanisms and transitions in railway wheel steels. Proc. IMechE part J: Journal of Engineering Tribology 218 (6) (2004) 467-478.

[30] T. Jendel, Prediction of wheel profile wear - comparisons with field measurements. Wear 253 (2002) 89-99.

[31] N. Tassini, X. Quost, R. Lewis, R. Dwyer-Joyce, C. Ariaudo, N. Kuka, A numerical model of twin disc test arrangement for the evaluation of railway wheel wear prediction methods. Wear 268 (2010) 660-667.

[32] W. Kik, J. Piotrowski, A fast, approximate method to calculate normal load at contact between wheel and rail and creep forces during rolling. Proceedings of the Second Mini-Conference on Contact Mechanics and Wear of Wheel/Rail Systems, ed. Zabory, TU Budapest (1996).

[33] F. Braghin, R. Lewis, R.S. Dwyer-Joyce, S. Bruni, A mathematical model to predict railway wheel profile evolution due to wear. Wear 261 (2006) 1253-1264.

[34] J. Sinclair, Friction Modifiers, in vehicle track interaction: identifying and implementing solutions. IMechE Seminar, February 17th, 2004.

[35] A.A. Shabana, K.E. Zaazaa, J.L. Escalona, J.R. Sany, Development of elastic force model for wheel/rail contact problems. Journal of Sound and Vibration 269 (2004) 295-325.

[36] R. Enblom, M. Berg, Simulation of railway wheel profile development due to wear - influence of disc braking and contact environment. Wear 258 (2005) 1055-1063.

[37] J. Tunna, J. Sinclair, J. Perez, A review of wheel wear and rolling contact fatigue. Proc. IMechE Vol. 221 Part F: J. Rail and Rapid Transit (2007) 271-289.

[38] S.A. Simson, C. Cole, Simulation of curving at low speed under high traction for passive steering locomotives. Vehicle System Dynamics 46 (2008) 1107-1121.

[39] A. Matsumoto, Y. Sato, H. Ohno, Y. Suda, Y. Michitsuji, M. Komiyama, N. Miyajima, M. Tanimoto, Y. Kishimoto, Y. Sato, T. Nakai, Curving performance evaluation for active-bogiesteering bogie with multibody dynamics simulation and experiment on test stand. Vehicle System Dynamics 46 Supp. (2008) 191-199.

[40] T.T. Vuong, P.A. Meehan, Wear transitions in a wear coefficient model. Wear 266 (2009) 898-906.

[41] S. Iwnicki, Handbook of Railway Vehicle Dynamics, CRC/Taylor \& Francis, 2006.

[42] V.K. Garg, R.V. Dukkipati, Dynamics of Railway Vehicle Systems, Academic Press Canada, 1984. 